\title{
Proximity Biotin Labeling Reveals KSHV Interferon Regulatory Factor Networks
}

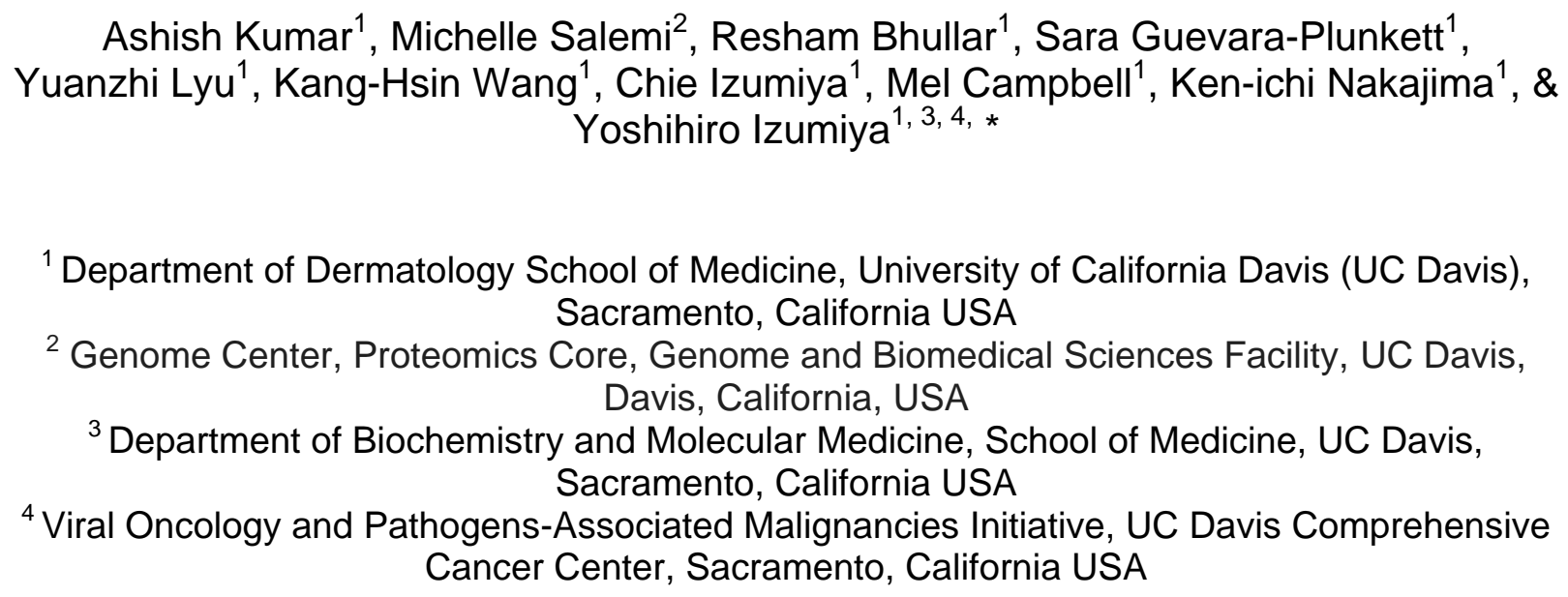

${ }^{4}$ Viral Oncology and Pathogens-Associated Malignancies Initiative, UC Davis Comprehensive Cancer Center, Sacramento, California USA

Short Title: Proximity Biotin Ligation for KSHV

*Correspondence: Yoshihiro Izumiya DVM, PhD E. mail: yizumiya@ucdavis.edu

Phone: 916-734-7253

Key words: KSHV, TurbolD, proteomics

Word count:

Abstract: 239

35 Importance: 102 


\section{Abstract}

37 Studies on "HIT\&RUN" effects by viral protein are difficult when using traditional affinity

38 precipitation-based techniques under dynamic conditions, because only proteins

39 interacting at a specific instance in time can be precipitated by affinity purification.

40 Recent advances in proximity labeling (PL) have enabled study of both static and

41 dynamic protein-protein interactions. Here we applied PL method with recombinant

42 Kaposi's sarcoma-associated herpesvirus (KSHV). KSHV, a gamma-herpesvirus,

43 uniquely encodes four interferon regulatory factors (IRFs 1-4) in the genome, and we

44 identified KSHV vIRF-1 and vIRF-4 interacting proteins during reactivation. Fusion of

45 mini-TurbolD with vIRF-1 or vIRF-4 did not interfere with KSHV gene expression, DNA

46 replication, or de novo infections. PL identified 213 and 70 proteins for vIRF-1 and VIRF-

474 respectively, which possibly interact during KSHV reactivation, and 47 of those were

48 shared between the two vIRFs; the list also includes three viral proteins, ORF17,

49 thymidine kinase, and vIRF-4. Functional annotation of respective interacting proteins

50 showed highly overlapping biological functions such as mRNA processing and

51 transcriptional regulation by TP53. Involvement of commonly interacting 44 cellular

52 proteins in innate immune regulation were examined by siRNAs, and we identified that

53 splicing factor 3B (SF3B) family proteins were clearly involved in interferons

54 transcription and suppressed KSHV reactivation. We propose that recombinant

55 TurbolD-KSHV is a powerful tool to probe key cellular proteins that play a role in KSHV

56 replication, and selective splicing factors may have a function beyond connecting two

57 exon sequences to regulate innate immune responses. 


\section{Importance}

60 Viral protein interaction with a host protein shows at least two sides: (i) taking host

61 protein functions for its own benefit and (ii) disruption of existing host protein complex

62 formation to inhibit undesirable host responses. Due to use of affinity-precipitation

63 approaches, the majority of our studies focused on how the virus takes advantage of the

64 newly-formed protein interactions for its own replication. Proximity labeling (PL)

65 however, can also highlight the transient and negative effects - those interactions which

66 lead to dissociation from the existing protein complex. Here we highlight the power of

$67 \mathrm{PL}$ in combination with recombinant KSHV to study viral host interactions. 


\section{Introduction}

69 Kaposi sarcoma herpesvirus (KSHV) is a pathogen associated with endothelial Kaposi's

70 sarcoma (KS) (1, 2), B-cell malignancies such as primary effusion lymphoma (PEL), and

71 AIDS related multicentric Castleman's disease (MCD) (3-6). In these cancer cells,

72 KSHV mostly exhibits latent infection, where most of the viral genes are silenced to

73 escape recognition by the host immune system. However, small population of infected

74 cells undergo spontaneous reactivation, where all of the KSHV genes are expressed for

75 production of progeny virions. Although lytic replication produces infectious virions and

76 facilitates transmission of the virus to neighboring cells or host, it also increases the risk

77 of the virus being caught by the host immune system (7). Host immune systems detect

78 pathogens through binding of pathogen associated molecular pattern (PAMPs) to

79 pattern recognition receptors (PRRs). Several PRRs such as IFI16 (8, 9), RIG-I (10-12),

80 TLR9 (13), TLR3 (14), TLR4 (15), and NLRP1 (16) are known to detect KSHV

81 associated PAMPs. The recognition of KSHV DNA by PRRs leads to phosphorylation,

82 dimerization, and nuclear translocation of IRF3/IRF7. IRF3/IRF7 binds to DNA through

83 its DNA binding domain (DBD), which results in secretion of cytokines and interferons

84 (IFN). To counteract the host response, KSHV encodes several immunomodulatory

85 proteins such as viral-interferon regulatory factors (vIRFs) that inhibit the antiviral

86 response and aid viral replication $(17,18)$.

87 KSHV genome encodes four vIRFs, vIRF-1-4. The N-termini of vIRFs exhibit similarity

88 to N-termini of cellular IRFs, however viral IRFs lack a key tryptophan residue, which is

89 required for binding to DNA (19). vIRF-1, vIRF-2, and vIRF-4 are inducible lytic genes,

90 although vIRF-1 can also be found in a small portion of latently infected cells. In contrast, 
91 vIRF-3 (also known as LANA2) was discovered as a latent protein and its expression

92 remains unchanged during reactivation (20). Studies on the function of vIRFs found that

93 vIRFs counteract the host IFN response by interacting with cellular proteins. vIRF-1

94 suppresses cellular IRF3-mediated transcription by binding to p300, thereby preventing

95 p300/CBP-IRF3 complex formation $(21,22)$. vIRF-1 also promotes KSHV lytic

96 replication by recruitment of USP7 (23). vIRF-2 was found to inhibit KSHV lytic gene

97 expression by increasing the expression of cellular antiviral factors like Promyelocytic

98 leukemia nuclear bodies (PML) (24). Similarly, vIRF-3 suppresses KSHV reactivation by

99 interacting with USP7, and the interaction also supports PEL cell growth (23).

100 Furthermore, vIRF-4 has been found to play a crucial role in triggering the KSHV

101 latency-to-lytic switch through interfering with the BCL6-vIRF-4 axis (25). vIRF-4 also

102 associates with IRF7, and inhibits IRF7 dimerization to suppress IFN production (26).

103 These studies sometimes showed different results in different cell lines, suggesting the

104 significance of implementing proteomic approaches that can reveal vIRFs interaction

105 networks more comprehensively. A broader view of the vIRFs interactomes will certainly

106 help to understand their diverse protein functions.

Dynamic and stable protein-protein interactions are key to cellular processes and

108 biological pathways. Affinity purification coupled with mass spectrometry (AP-MS) has

109 been an invaluable method used to identify protein-protein interactions. However, AP-

110 MS often fails to identify weakly or transiently interacting proteins. To overcome this

111 drawback, enzyme-based Proximity-based labeling (PL) approaches have been

112 developed. The approach provides sensitivity and specificity required to study dynamic 113 protein-protein interaction. BirA $A_{R 118 G}(B i r \mid D)$ was the first proximity-based labelling 
114 enzyme identified in E.coli which conjugates biotin to lysine residues of neighboring

115 proteins (27). However, original BirlD required the presence of biotin for several hours

116 to be able to biotinylate a sufficient amount of proteins for analysis, thereby restricting

117 its use for dynamic processes. Recently, two variants of BirlD have been developed by

118 directed evolution named as mini-TurbolD (28 kD) and TurbolD (35 kD), which allow

119 proximity labeling in less than $10 \mathrm{~min}$ without significant toxicity (28). The TurbolD

120 based approach has already been successfully employed in a wide variety of species

121 including mammalian cells (28-32), Drosophila (33), plants (34-37), yeast (38), flies and

122 worms (28). In this study, we prepared recombinant 3xFlag-mini-TurbolD-vIRF-1 and

123 3xFlag-mini-TurbolD-vIRF-4 KSHV that employs mini-TurbolD to biotinylate host and

124 viral proteins in vicinity to these two viral proteins. The proximity-labeling approach

125 combined with mass spectrometry identified both previously-identified cellular proteins,

126 as well as new host proteins as their interacting partners. The siRNA screenings of

127 these interacting proteins identified that selective splicing factors function to suppress

128 KSHV reactivation and are associated with anti-viral responses. 


\section{$130 \quad$ Materials and Methods}

\section{Chemicals}

132 Dulbecco's modified minimal essential medium (DMEM), Fetal bovine serum (FBS), 133 phosphate buffered saline (PBS), Trypsin-EDTA solution, 100x Penicillin-streptomycin-

134 L-Glutamine solution and Strep-HRP conjugate were purchased from Thermo Fisher 135 (Waltham, MA USA). Puromycin and G418 solution were obtained from InvivoGen (San 136 Diego, CA, USA). Hygromycin B solution was purchased from Enzo Life Science 137 (Farmingdale, NY, USA). Anti-ORF57, anti-K8, and anti-K8.1, antibodies were 138 purchased from Santa Cruz Biotechnology Inc (Santa Cruz, CA, USA). Anti-K-Rta 139 antibody was described previously (39). All other chemicals were purchased from 140 Millipore-Sigma (St. Louis, MO, USA) unless otherwise stated.

\section{Cells, siRNA transfection and reagents}

143 iSLK.219 cells were maintained in DMEM medium supplemented with 10\% FBS, 10

$144 \mu \mathrm{g} / \mathrm{ml}$ puromycin, $400 \mu \mathrm{g} / \mathrm{ml}$ hygromycin $\mathrm{B}$, and $250 \mu \mathrm{g} / \mathrm{ml} \mathrm{G} 418$. iSLK cells were 145 maintained in DMEM medium supplemented with 10\% FBS, 1\% penicillin-streptomycin 146 solution and $10 \mu \mathrm{g} / \mathrm{ml}$ puromycin. iSLK cells were obtained from Dr. Don Ganem 147 (Novartis Institutes for Biomedical Research). A549 cells were obtained from Dr. 148 Tsucano (University of California, Davis). A549 cells were grown in DMEM containing $14910 \%$ FBS and 1\% penicillin-streptomycin. Transfection of siRNA in iSLK.219 cells was 150 performed with Lipofectamine RNAiMax reagent (Invitrogen) according to 151 manufacturer's protocol. 


\section{Quantification of viral replication}

154 siRNA targeting the cellular genes were transfected in iSLK.219 cells for $48 \mathrm{~h}$ followed

155 by KSHV reactivation by doxycycline $(1 \mu \mathrm{g} / \mathrm{ml})$. After $24 \mathrm{~h}$, the RFP fluorescence 156 intensity was quantified using ImageJ software. The RFP signal intensity was 157 normalized relative to non-targeting siRNA (NTC).

159 Construction of vIRF-1 and vIRF-4 miniTurbo KSHV BAC16

160 Recombinant KSHV was prepared by following a protocol for En passant mutagenesis 161 with a two-step markerless red recombination technique (40). Briefly, codon optimized 162 mini-TurbolD coding sequence (Table 1), which also encodes $3 x$ Flag tag was first 163 cloned into a pBS SK vector (Thermo Fisher, Waltham, MA USA). The pEPkan-S 164 plasmid was used as a source of the kanamycin cassette, which includes the I-Secl 165 restriction enzyme site at the 5'-end of kanamycin coding region (40). Kanamycin 166 cassette was amplified with primer pairs listed in Table 1, and cloned into the mini167 TurbolD coding region at a unique restriction enzyme site. The resulting plasmid was 168 used as a template for another round of PCR to prepare a transfer DNA fragment for 169 markerless recombination with BAC16 (41). Recombinant BAC clones with insertion 170 and also deletion of the kanamycin cassette in the BAC16 genome were confirmed by 171 colony PCR with appropriate primer pairs. Recombination junctions and adjacent 172 genomic regions were amplified by PCR and the resulting PCR products were directly 173 sequenced with the same primers to confirm in-flame insertion of mini-TurbolD cassette 174 into the BAC DNA. The resulting recombinant BAC was confirmed by restriction enzyme 175 digestions (Hindll and Bg/lI), if there were any large DNA deletions. Two independent 
176 BAC clones were generated for each mini-TurbolD tagged recombinant virus as

177 biological replicates, and used one of the clone for protein ID. Entire BAC DNAs were

178 subsequently sequenced.

\section{Western blotting}

181 Cells were lysed in IP lysis buffer (25 mM Tris-HCl pH 7.4, 150 mM NaCl, 1\% NP-40, 1

$182 \mathrm{mM}$ EDTA, 5\% glycerol) containing protease inhibitors (Roche, Basel, Switzerland).

183 Total cell lysates $(25 \mu \mathrm{g})$ were boiled in SDS-PAGE loading buffer and subjected to 184 SDS-PAGE and subsequently transferred to a polyvinylidene fluoride membrane 185 (Millipore-Sigma, St. Louis, MO, USA) using a semidry transfer apparatus (Bio-Rad, 186 Hercules, CA, USA). Streptavidin-HRP conjugate was used at 1:3000 dilution. Final 187 dilution of the primary antibody was $1: 5,000$ for anti-K-Rta rabbit serum, $1 \mu \mathrm{g} / \mathrm{mL}$ of anti$188 \mathrm{~K} 8 \alpha$ (Santa Cruz, Santa Cruz, CA, USA), $1 \mu \mathrm{g} / \mathrm{mL}$ of anti-ORF57 mouse monoclonal 189 antibody (Santa Cruz, Santa Cruz, CA, USA), $1 \mu \mathrm{g} / \mathrm{mL}$ of anti-K8.1 mouse monoclonal 190 (Santa Cruz, Santa Cruz, CA, USA), and 1:5,000 for anti- $\beta$-actin mouse monoclonal 191 (Millipore-Sigma, St. Louis, MO, USA). Washing membranes and secondary antibody 192 incubations were performed as described previously (42).

\section{Quantification of viral copy number}

195 Two hundred microliter of cell culture supernatant was treated with $12 \mu \mathrm{g} / \mathrm{ml}$ of DNase I

196 for $15 \mathrm{~min}$ at room temperature to degrade uncapsidated DNA. This reaction was 197 stopped by the addition of EDTA to $5 \mathrm{mM}$ followed by heating at $70^{\circ} \mathrm{C}$ for $15 \mathrm{~min}$. Viral 198 genomic DNA was purified using QIAamp DNA Mini Kit according to the manufacturer's 
199 protocol, and eluted in $100 \mu \mathrm{l}$ of buffer AE. Four microliters of eluate was used for real-

200 time qPCR to determine viral copy number, as described previously (42).

202 Preparation of purified KSHV

203 iSLK cells latently infected with mini-TurbolD-KSHVs were seeded in eight to ten $15 \mathrm{~cm}$ 204 dishes, and stimulated with $1 \mu \mathrm{g} / \mathrm{mL}$ of doxycycline and $3 \mathrm{mM}$ sodium butyrate $(\mathrm{NaB})$ for $20524 \mathrm{~h}$ and further incubated with culture media without stimuli for $72 \mathrm{~h}$. The culture 206 supernatant was centrifuged using the Beckman SW28 rotor $(25,000 \mathrm{rpm}$, for 2 h) with $20725 \%$ sucrose cushion. Virus pellet was dissolved in DMEM and further purified by 208 discontinuous sucrose gradient (25-60\%) centrifugation using Beckman SW40Ti rotor 209 (21,000 rpm, for $16 \mathrm{~h})$. The virus pellet was dissolved in DMEM for infection.

211 Real time RT-PCR

212 Total RNA was isolated using Quick-RNA miniprep kit (Zymo Research, Irvine, CA, 213 USA). First strand cDNA was synthesized using High Capacity cDNA Reverse 214 Transcription Kit (Thermo Fisher, Waltham, MA USA). Gene expression was analyzed 215 by realtime qPCR using specific primers for KSHV ORFs designed by Fakhari and 216 Dittmer (43). We used $18 \mathrm{~S}$ ribosomal RNA as an internal standard to normalize viral 217 gene expression.

\section{Affinity purification of biotinylated proteins}

220 The affinity purification was done with streptavidin coated magnetic beads (Thermo-

221 Fisher). Briefly, $150 \mu$ magnetic beads/sample were pre-washed with RIPA lysis buffer 
222 (150 mM NaCl, 5 mM EDTA (pH 8), 50 mM Tris (pH 8), 1\% NP-40, 0.5\% sodium

223 deoxycholate, $0.1 \%$ SDS) 3 times. Total $3 \mathrm{mg}$ of whole cell lysate was incubated with

224 pre-washed streptavidin beads at room temperature for $1 \mathrm{~h}$ for rotation. The beads were

225 collected using magnetic stand and washed three times with wash buffer according to

226 manufacturer's protocol. Finally, beads were resuspended in $200 \mu$ of wash buffer and

227 sent to UC Davis Proteomics core for on bead digestion and LC-MS/MS analysis.

229 MS sample preparation

230 Protein samples on magnetic beads were washed four times with $200 \mu$ of $50 \mathrm{mM}$

231 ammonium bicarbonate (AMBIC) with a twenty-minute shake time at $4^{\circ} \mathrm{C}$ in between

232 each wash. Roughly $2.5 \mu \mathrm{g}$ of trypsin was added to the bead and AMBIC and the

233 samples were digested over night at $800 \mathrm{rpm}$ shake speed. After overnight digestion,

234 the supernatant was removed, and the beads were washed once with enough $50 \mathrm{mM}$

235 ammonium bicarbonate to cover. After 20 minutes at a gentle shake the wash is

236 removed and combined with the initial supernatant. The peptide extracts are reduced in

237 volume by vacuum centrifugation and a small portion of the extract is used for

238 fluorometric peptide quantification (Thermo scientific Pierce). One microgram of sample

239 based on the fluorometric peptide assay was loaded for each LC-MS analysis.

240 Digested peptides were analyzed by LC-MS/MS on a Thermo Scientific Q Exactive

241 Orbitrap Mass spectrometer in conjunction Proxeon Easy-nLC II HPLC (Thermo

242 Scientific) and Proxeon nanospray source. The digested peptides were loaded a 100

243 micron x $25 \mathrm{~mm}$ Magic C18 $100 \AA 5 \mathrm{U}$ reverse phase trap where they were desalted

244 online before being separated using a 75 micron x $150 \mathrm{~mm}$ Magic C18 $200 \AA 3 \mathrm{U}$ reverse 
245 phase column. Peptides were eluted using a 60-minute gradient with a flow rate of 300

$246 \mathrm{nl} / \mathrm{min}$. An MS survey scan was obtained for the $\mathrm{m} / \mathrm{z}$ range $300-1600$, MS/MS spectra

247 were acquired using a top 15 method, where the top 15 ions in the MS spectra were

248 subjected to HCD (High Energy Collisional Dissociation). An isolation mass window of

$2492.0 \mathrm{~m} / \mathrm{z}$ was for the precursor ion selection, and normalized collision energy of $27 \%$ was

250 used for fragmentation. A fifteen second duration was used for the dynamic exclusion.

\section{MS/MS analysis}

253 Tandem mass spectra were extracted and charge state deconvoluted by Proteome

254 Discoverer (Thermo Scientific) All MS/MS samples were analyzed using X! All MS/MS

255 samples were analyzed using X! Tandem (The GPM, thegpm.org; version X! Tandem

256 Alanine (2017.2.1.4)). X! Tandem was set up to search the Human and Kaposi Sarcoma

257 Herpes virus database (149182 entries) assuming the digestion enzyme trypsin. X!

258 Tandem was searched with a fragment ion mass tolerance of 20 PPM and a parent ion

259 tolerance of 20 PPM. Carbamidomethyl of cysteine and selenocysteine was specified in

$260 \mathrm{X}$ ! Tandem as a fixed modification. Glu->pyro-Glu of the N-terminus, ammonia-loss of

261 the N-terminus, gln->pyro-Glu of the N-terminus, deamidated of asparagine and

262 glutamine, oxidation of methionine and tryptophan and dioxidation of methionine and

263 tryptophan were specified in X! Tandem as variable modifications.

264 Scaffold (version Scaffold_4.8.4, Proteome Software Inc., Portland, OR) was used to

265 validate MS/MS based peptide and protein identifications. Peptide identifications were

266 accepted if they could be established at greater than $98.0 \%$ probability by the Scaffold

267 Local FDR algorithm. Peptide identifications were also required to exceed specific 
268 database search engine thresholds. Protein identifications were accepted if they could

269 be established at greater than $5.0 \%$ probability to achieve an FDR less than $5.0 \%$ and

270 contained at least 2 identified peptides. Protein probabilities were assigned by the

271 Protein Prophet algorithm (44). Proteins that contained similar peptides and could not

272 be differentiated based on MS/MS analysis alone were grouped to satisfy the principles

273 of parsimony. Proteins sharing significant peptide evidence were grouped into clusters.

274

275 Pathway analysis

276 The proteins identified to be interacting with vIRF-1 and vIRF-4 were used for Gene

277 ontology and network analysis. The top gene ontology processes were enriched by

278 Metascape web-based platform, and the Metascape software was used for gene 279 ontology and network analysis (45).

\section{Statistical analysis}

282 Results are shown as mean \pm SD from at least three independent experiments. Data 283 was analyzed using unpaired Student's t test, or ANOVA followed by Tukey's HSD test.

284 A value of $p<0.05$ was considered statistically significant. 


\section{Results}

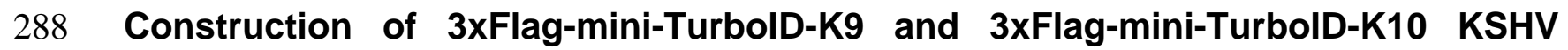

289 BAC16. Biotin labelled proximity labeling $(P L)$ has emerged as a powerful method for 290 probing various target proteins in a wide variety of species including mammalian cells 291 and unicellular organism $(28,32,33,35,37,38)$. We thought that applying the 292 technique to virology would be particularly beneficial, because not only viral-host 293 interactions are inherently dynamic but viruses are also completely dependent on host 294 cell machinery for their replication. In fact, many key cellular proteins, such as p53, were 295 identified from virology as viral protein interacting proteins. Our major goal is thus to 296 report on the successful application and utility of PL in conjunction with recombinant 297 KSHV BAC system.

To generate recombinant KSHV conveniently, we first prepared a template 299 plasmid, which is used to create PCR fragments for recombination. The template 300 encodes a 3xFlag tag at the N-terminus of mini-TurbolD and kanamycin cassettes in 301 mini-TurbolD coding region as an excisable format with I-Secl induction. The 3xFlag302 mini-TurbolD-kana DNA fragment was amplified with primers with homology arms, and 303 amplified fragments were then used for recombination by using a two-step 304 recombination approach as previously described (40) (Figure 1A). The 3xFlag-mini305 TurbolD-K9 and 3xFlag-mini-TurboID-K10 BAC16 were directly transfected into iSLK 306 cells and selected with hygromycin $(1 \mathrm{mg} / \mathrm{ml})$ to generate iSLK cells harboring latent 307 3xFlag-miniTurbolD-K9 KSHV genome (named as vIRF-1 mini-TurbolD cells) and 308 3xFlag-mini-TurboID-K10 KSHV (named as vIRF-4 mini-TurbolD cells) (Figure 1B). 
Next, optimal concentration of exogenous biotin and duration of incubation time

310 for efficient labelling was determined. The vIRF-1 and vIRF-4 mini-TurbolD cells were

311 reactivated for $24 \mathrm{~h}$, incubated with varying concentration of biotin $(0,125,250$ and 500

$312 \mu \mathrm{M}$ ) for $1 \mathrm{~h}$, and subsequently monitored for their biotinylation signal in whole cell

313 lysates using streptavidin immunoblots. Untreated cells in absence of biotin were used

314 as negative control. Immunoblot analysis using the HRP-conjugated streptavidin

315 showed multiple biotinylated protein indicating successful labelling of proteins with vIRF-

3161 and vIRF-4 tagged mini-TurboID. Comparable levels of signal intensity were observed

317 until $500 \mu \mathrm{M}$, suggesting that saturation of protein biotinylation occurs at $125 \mu \mathrm{M}$

318 (Figure 1C). Similarly, vIRF-1 Turbo and vIRF-4 mini-TurbolD cells were incubated with

319 biotin for various time periods. Biotinylation signal was seen within 15 mins after

320 addition of exogenous biotin and streptavidin signals gradually increased along with

321 incubation time (Figure 1D). Considering only a small proportion of cells were

322 reactivating in a dish, we concluded that there was a sufficient amount of biotinylation in

323 the cells for protein identification. For following studies, we decided to use a saturating

324 amount of biotin $(500 \mu \mathrm{M})$ for 60 mins incubation.

326 Gene expression in vIRF-1 and vIRF-4 mini-TurbolD cells. We next verified the

327 induction of viral genes to ensure that tagging $\mathrm{K} 9$ or $\mathrm{K} 10$ gene with 3xFlag-mini-TurbolD

328 has little effects on viral gene expression and replication. For this, we stimulated vIRF-1

329 and vIRF-4 mini-TurbolD cells with Doxycycline (Dox) and performed qPCR for selected

330 KSHV genes. We observed induction of KSHV lytic genes, PAN RNA, ORF6, vIRF-1

331 and vIRF-4 (Figure 2A). In addition, we verified induction of selected lytic KSHV 
332 proteins at $48 \mathrm{~h}$ (Figure $\mathbf{2 B}$ ), and virion production in culture supernatant at $96 \mathrm{~h}$ post-

333 reactivation. Finally, culture supernatant was also used to infect A549 recipient cells to

334 verify infectivity (Figure 2C). Altogether, these observations indicate that 3xFlag-mini-

335 TurbolD protein tag did not interfere with viral gene expression and that the recombinant

336 KSHVs are replicating to produce infectious viral particles.

338 Proximity biotin labelling with vIRF-1 and vIRF-4. For proximity protein labeling,

339 three replicated samples were prepared for both vIRF-1 and vIRF-4 mini-TurbolD cells.

340 Cells were reactivated with Doxycycline and NaB (sodium butyrate) for $24 \mathrm{~h}$ followed by

341 addition of biotin for $1 \mathrm{~h}$. Two sets of controls were also processed concurrently, in order

342 to rule out non-specific precipitations. In the first set, the cells were left without triggering

343 reactivation followed by incubation with biotin $(+B)$ to rule out non-specific protein

344 binding with biotin (Ctrl 1). For the second set, cells were reactivated with Dox/TPA for

$34524 \mathrm{~h}$ and incubated for additional $1 \mathrm{~h}$ in the absence of biotin (-B) to rule out non-

346 specific interaction with streptavidin beads (Ctrl 2). Schematic workflow for the

347 experiment is presented in Figure $\mathbf{3 A}$. We confirmed the biotinylation signal by

348 streptavidin blot, and vIRF-1 and vIRF-4 expression by using anti-Flag antibody (Figure

349 3B). The whole cell lysate from vIRF-1 and vIRF-4 mini-TurbolD cells were further used

350 for enrichment of biotinylated protein using magnetic beads coated with streptavidin.

351 The enriched proteins were eluted from the streptavidin beads using trypsin on-bead

352 digestion overnight. Ctrl1 and ctrl2 were used independently to remove background

353 noise. We designated proteins with $p$-value $<0.05$ and fold change $>2$ over both ctrl 1 
354 and ctrl2 as positive hits. Based on our setting, we identified 213 and 70 proteins from

355 vIRF-1 and vIRF-4 mini-TurbolD cells respectively (S-Table 1, S-Table 2).

357 vIRF-1 and vIRF-4 pathway analysis.

358 Next, gene ontology (GO) analysis was performed for proteins identified in vIRF-1 and

359 vIRF-4 mini-TurbolD cells. The vIRF-1 interactome revealed significant enrichment for

360 functions related to mRNA processing, transcription regulation by TP53, regulation of

361 mRNA processing, and formation of RNA pol II elongation complex. Top 20 enriched

362 GO terms are presented in Figure 4A (upper panel). Similarly, GO analysis for the

363 vIRF-4 revealed again enrichment of mRNA processing, regulation of mRNA processing,

364 mRNA polyadenylation, and mRNA splicing [Figure 4A (lower panel)]. Consistent with

365 the fact that vIRF-1 and vIRF-4 have overlapping biological functions, we found

366 overlapping possible pathway regulations in vIRF-1 and VIRF-4. Network plot by

367 Cytoscape was generated using a subset of enriched proteins to highlight their

368 respective protein networks (Figure 4B).

370 Effects of common hits in KSHV replication.

371 Previous studies demonstrated that vIRF-1 and vIRF-4 possess similar biological

372 functions to regulate interferon pathways $(18,20)$. We thus hypothesize that commonly

373 targeted cellular proteins by the two viral proteins play an important role in interferon

374 responses. Our venn diagram indicated 123 and 23 proteins were interacting

375 exclusively with vIRF-1 and vIRF-4, respectively, and 47 proteins were found to be

376 interacting with both VIRF-1 and VIRF-4. This suggests that the majority of vIRF-4 
377 interacting proteins (67\%) are also neighbors to vIRF-1 (Figure 5A). Of the 47 proteins

378 interacting with both vIRF-1 and vIRF-4, 44 were cellular proteins whereas 3 were viral

379 proteins (Figure 5A). To examine the role of those cellular proteins in KSHV replication,

380 iSLK.219 cell line was employed. iSLK.219 carries a recombinant rKSHV.219 virus

381 encoding a constitutively expressing GFP and an PAN RNA promoter driven RFP

382 reporter in the viral genome, allowing us to monitor the lytic promoter activation. We

383 used siRNA to knock-down these 44 cellular proteins followed by KSHV reactivation by

384 treatment with Dox to induce K-Rta expression. We found that knock-down of 17 genes

385 enhanced KSHV promoter activation, while knock-down of 6 genes lowered KSHV gene

386 transactivation (Figure 5B). The corresponding images of selected knock-down

387 experiments are shown in Figure 5C, and the results were further confirmed by

388 quantifying the viral mRNAs after knock-down of selected genes, SF3B1, SF3B2 and

389 SNW1 (Figure 5D). Consistent with increased viral gene expression, the viral DNA copy

390 number in culture supernatant was increased by knocking-down of SF3B1, SF3B2 or

391 SNW1 (Figure 5E). Taken together, our study suggests that some splicing factors have

392 a role in restricting KSHV gene expression during reactivation, albeit their biological

393 roles in general host gene transcription.

Splicing factor 3B (SF3B) subunits are important for IFN gene expression.

396 Previous reports showed that the KSHV genome is sensed by RIG-I like receptors.

397 Polyl:C is a synthetic dsRNA polymer which is recognized by RIG-I, leading to strong

398 induction of interferons and interferon stimulatory genes (ISGs). Because KSHV vIRFs

399 are known counteract IFN responses, we examined the relation of SF3B1 and SNW1 to 
400 interferon responses with polyl:C. The results showed that knock-down of SF3B1 or

401 SNW1 clearly inhibited induction of type I interferon (IFNB1), type III interferon (IFNL1),

402 and interferon downstream target gene (DDX58) [Figure 6 (a-c)], but not the non-IFN

403 regulatory gene [Figure 6(d)].

404

\section{Discussion}

406 Since viral replication depends entirely upon host factors, understand the virus and host 407 protein interaction network is important to find their Achilles's heel (46). We therefore 408 applied a mini-TurbolD based system for studying the virus and host protein interaction. 409 By constructing mini-TurbolD as an integral component of KSHV BAC16 recombination 410 system, we demonstrated a novel approach to define protein interaction networks. We 411 propose that this approach increases the reproducibility of identifying interacting 412 proteins, because tight interaction between biotin and streptavidin allows us to wash

413 magnetic beads in highly stringent conditions to remove non-specific or indirect protein 414 interactions. High reproducibility could be seen in our biological triplicated samples (S415 Fig. 1).

416 To conveniently generate mini-TurbolD tagged viruses, we first generated 417 template plasmids similar to what we did for Rainbow-KSHV (47). With a plasmid 418 template, homology arms can be added to primer pairs and the resultant PCR product is 419 used for recombination (Fig. 1A). Background BAC16 can also be wild type BAC16, 420 mutant virus, and/or Rainbow-KSHV, which allows us to examine the formation of 421 protein complexes during viral replication and the effects of specific mutations. In this 422 study, we used vIRF-1 and vIRF-4 as bait for validating the efficiency of PL. The vIRF-1 
423 and vIRF-4 were selected because of their known role in regulation of innate immune

424 response during $\mathrm{KSHV}$ reactivation, and multiple interacting proteins have been

425 identified that can be used as comparisons $(18,23,26,48)$. Consistent with previous

426 studies, vIRF-1 and vIRF-4 were found to be physically neighboring to cellular proteins

427 that function in p53 transcriptional regulation. vIRF-1 was reported to deregulate p53

428 activity by interacting with ATM kinase and prevent serine 15 phosphorylation (49). In

429 addition, vIRF-1 interacts directly with p53 to inhibit its transcriptional activation (48).

430 Although our studies could not precipitate p53, we identified p53BP1 (p53 binding

431 protein 1) as a possible partner of vIRF-1. We could also identify USP7 in both vIRF-1

432 and vIRF-4 samples, validating the PL approaches (23).

433 After learning that mini-Turbo worked efficiently in biotinylating cellular proteins,

434 we tagged various other KSHV genes with mini-TurboID using the same approach.

435 However, we learned that efficacies of biotin labeling varies significantly among different

436 viral proteins. For example, mini-Turbo-ORF57 robustly induced biotinylated protein in

437 total lysates with as little as 15 min of D-biotin incubation, while biotinylation by mini-

438 Turbo-ORF50 was barely detectable in the same time frame. For this study, we also

439 generated VIRF-2 and VIRF-3 constructs at same time; however, the level of

440 biotinylation was lower with the same amount of D-biotin and incubation periods,

441 leading us to drop these analyses for comparison. Differences in efficacy of biotinylation

442 have also been seen in prior studies and abundance of viral protein expression during

443 reactivation and subcellular nuclear localization seemed to have strong effects in the

444 outcome of biotinylation. 
Our PL studies showed a large portion of host proteins $(36 \%)$ were related to

446 mRNA processing. Within these RNA processing proteins, SF3B1, SF3B2 and SF3B3,

447 a component of SF3b complex, were clear front runners for our further analyses. The

448 SF3b complex is a component of the functional U2 small nuclear ribonucleoprotein

449 (snRNP), which recognizes the exon/intron junctions and facilitates spliceosome

450 assembly (50). Even though SF3B1 is one of many cellular genes involved in RNA

451 splicing, SF3B1 has been specifically identified as a commonly mutated gene in

452 myelodysplastic syndrome (MDS) at 25-30\% frequencies in MDS patients (51-53).

453 Recent studies also showed that SF3B1 mutations increase R-loop formation and DNA

454 damage (54). Here we found SF3B1 knock-down inhibited IFN gene expression 3 to 4-

455 fold and also enhanced KSHV reactivation. In fact, SF3A1 and SF3B1 were reported to

456 play a role in innate immune response to TLR ligands. The study showed that SF3A1

457 and SF3B1 are necessary to increase production of IL-6 and IFN $\beta$ by modulating the

458 splicing of MyD88, an important adaptor molecule for TLR signaling pathway (55).

459 Based on that study and ours, we propose that targeting the splicing complex might be

460 a previously uncharacterized mechanism for KSHV to modulate host immune responses.

461 Further studies on regulation of SF3B complex formation during KSHV reactivation

462 and/or IFN stimulation with PL will clarify underlying mechanisms of SF3B family

463 proteins in KSHV replication and IFN regulation.

464 In addition to SF3 complex, several other mRNA processing factors like XAB2,

465 SNRPD1, SNW1, RBM10, SYMPK, and GTF2F2 were found to suppress KSHV

466 reactivation (Fig 5). A recent study showed that SNW1 interacts with IKKy, the

467 regulatory subunit of IKB kinase (IKK) complex. SNW1 increases production of IL-6, 
468 IFN $\beta$, and $\mathrm{MX} 1$ by enhanced activation of NF-KB and phosphorylation of TBK1 in 469 response to influenza A virus and polyl:C (56). Influenza A virus and polyl:C are

470 recognized by the innate immune sensor RIG-I, which plays an important role in

471 suppressing KSHV reactivation by sensing $\operatorname{KSHV}$ DNA (11, 12, 57, 58). Accordingly, we

472 explored the role of SNW1 in regulating RIG-I mediated innate immune response during

473 KSHV reactivation. We found that knock-down SNW1 indeed enhanced KSHV 474 replication (Fig. 5D), and this effect could be through down-regulation of IFNß (Fig. 6).

475 In summary, using mini-TurbolD KSHV with vIRFs as bait, we could successfully 476 probe cellular proteins that play a role in innate immune responses. We propose mini-

477 TurbolD with recombinant KSHV BAC system as a very powerful combination to identify 478 cellular proteins that play an important role in KSHV replication, hence a key player for 479 respective cellular pathways.

\section{Acknowledgement}

482 We would like to thank all members in Izumiya lab for valuable discussion and 483 assistances. This research was supported by public health grants from National Cancer 484 Institute (CA225266, CA232845), National Institute of Dental and Craniofacial 485 (DE025985), and National Institute of Allergy and Infectious Disease (Al147207) to Y.I. 


\section{$487 \quad$ Figure Legends}

488 Figure 1. Engineering of mini-TurbolD KSHVs. (A) Schematic diagram for construction of 3xFlag-miniTurbolD-K9 and 3xFlag-miniTurbolD-K10 KSHV

490 BAC16. (i) The codon optimized cDNA fragment (900 bp) of mini-TurbolD was 491 synthesized and cloned into pBS vector between Kpnl and Sacll restriction enzyme 492 sites. (ii) The kanamycin cassette with I-Scel recognition sequence along with 50 bp 493 homologous sequence was generated by PCR with pEP-Kan plasmid as a template, 494 and cloned into Accl restriction enzyme site. (iii-v) The resulting plasmid was fully 495 sequenced and used as a template to generate a DNA fragment for homologous 496 recombination with BAC16 inside bacteria. (vi, vii) After confirmation of insertion at 497 correct site by colony PCR screening, the kanamycin cassette was deleted by 498 recombination with induction of I-Scel in bacteria by incubating with L-Arabinose. 499 Correct insertion of the mini-TurbolD and integrity of BAC DNA were confirmed by 500 sequencing of PCR-amplified fragments and restriction digestions. Primers and DNA

501 fragment used are listed in Table 1. (B) Generation of vIRF-1 and vIRF-4 TurbolD 502 stable cells. iSLK cells were transfected with 3xFlag-miniTurbolD-K9 and 3xFlag503 miniTurbolD-K10 KSHV BAC16 and stably selected with hygromycin (1 mg/ml). GFP 504 images show iSLK latently infected with 3xFlag-miniTurbolD-K9 (upper panels) and 505 3xFlag-miniTurbolD-K10 KSHV BAC16 (lower panels). BF: Bright Field, GFP: Green 506 fluorescent protein. (C) Biotin ligase activity of mini-TurbolD tagged vIRF-1 and 507 vIRF-4. The vIRF-1 and vIRF-4 mini-TurbolD cells were stimulated with Dox $(1 \mu \mathrm{g} / \mathrm{ml})$ 508 and $\mathrm{NaB}(3 \mathrm{mM})$ for $24 \mathrm{~h}$ followed by incubation with indicated concentration of $\mathrm{D}$-biotin 509 for $1 \mathrm{~h}$. Activity of mini-TurbolD was examined by immunoblot using Streptavidin HRP 
510 conjugate. WB: Western Blot. (D) Dependency of mini-TurbolD on labelling time.

511 vIRF-1-Turbo and vIRF-4 mini-TurbolD cells were stimulated with Dox $(1 \mu \mathrm{g} / \mathrm{ml})$ and

$512 \mathrm{NaB}(3 \mathrm{mM})$ for $24 \mathrm{~h}$ followed by incubation with D-biotin $(500 \mu \mathrm{M})$ for indicated time-

513 points. Activity of mini-TurbolD was analyzed by immunoblot using Streptavidin HRP

514 conjugate. WB: Western Blot, m: minutes, h: hours.

516 Figure 2. Viral gene expression and production of progeny virus. (A) Viral gene

517 expression for vIRF-1 and vIRF-4 mini-TurbolD cells. The vIRF-1 and vIRF-4 mini-

518 TurbolD cells were stimulated with Dox $(1 \mu \mathrm{g} / \mathrm{ml})$ for 24 and $48 \mathrm{~h}$. Total RNA was

519 purified at indicated time point and subjected to real-time PCR for indicated genes.

520 Gene expression is shown as a $2^{-\Delta C T}$. $18 \mathrm{~S}$ ribosomal RNA was used as an internal

521 standard for normalization. (B) Viral protein expression in vIRF-1 and vIRF-4 mini-

522 TurbolD cells. The vIRF-1 and vIRF-4 mini-TurbolD cells were stimulated with Dox (1

$523 \mu \mathrm{g} / \mathrm{ml})$ and $\mathrm{NaB}(3 \mathrm{mM})$ for $24 \mathrm{~h}$. Total cell lysates were subjected to immunoblotting

524 using KSHV proteins and $\beta$-actin protein specific antibodies. (C) De novo infection.

525 A549 cells were infected with vIRF-1 and vIRF-4 mini-TurbolD virus. BF: Bright field, 526 GFP: Green fluorescent protein.

528 Figure 3. Proteins in close-proximity to vIRF-1 and vIRF-4. (A) Schematic 529 workflow for experimental setup. Three biological replicates for each sample were 530 analyzed by LC-MS/MS analysis. The plus (+) and minus (-) signs indicate presence 531 and absence, respectively. Ctrl: Control, Expt: Experimental, D/N: Dox $(1 \mu \mathrm{g} / \mathrm{ml})$ and $532 \mathrm{NaB}(3 \mathrm{mM})$. (B) Confirmation of biotinylation. The cell lysate from one of the three 
533 biological replicates was subjected to immunoblotting using streptavidin HRP

534 conjugates, Flag antibody and $\beta$-actin antibody. WB: Western Blotting. (C-D)

535 Identification of proteins in close proximity to vIRF-1 and vIRF-4. Volcano plot

536 showing differential proteins profiles in Ctrl 1 and Expt, and Ctrl 2 and Expt for vIRF-1

537 (C) and vIRF-4 mini-TurbolD expressing cells (D). Identified and quantified biotinylated

538 peptides are plotted as log2 fold change (Expt/Ctrl1) or (Expt/Ctrl2) versus -log10 p-

539 value. Biotinylated peptide for vIRF-1 (C) and vIRF-4 (D) are shown with red arrow.

540 Yellow boxes indicate selected peptides with fold change $>2$ and $p$ value $<0.05$. (E)

541 Venn diagram comparing proteomic lists between Ctrl1 vs Expt and Ctrl2 vs Expt (left

542 panel for vIRF-1 and right panel for vIRF-4).

544 Figure 4. Pathway analysis for vIRF-1 and vIRF-4 interacting protein. (A) Top non-

545 redundant enrichment clusters for vIRF-1 (top panel) and vIRF-4 (bottom panel)

546 interacting proteins using Metascape bar graph (30944313). Color scales represent

547 statistical significance. (B) Metascape enrichment network visualization for vIRF-1 (top

548 panel) and vIRF-4 (bottom panel) showing the intra-cluster and inter-cluster similarities

549 of enriched terms, up to ten terms per cluster. Cluster annotations are shown in color

550 code.

552 Figure 5. Splicing factor 3B (SF3B) subunits are suppressors for KSHV

553 reactivation. (A) Common protein between vIRF-1 and vIRF-4. Venn diagram

554 depicting proteins that interact with vIRF-1 and vIRF-4. List of cellular proteins and viral

555 protein interacting with $\mathrm{VIRF}-1$ and $\mathrm{VIRF}-4$. (B) KSHV reactivation. Five pmol of 
556 individual siRNAs were transfected into iSLK.r219 cells for $48 \mathrm{~h}$ followed by reactivation

557 with Dox $(1 \mu \mathrm{g} / \mathrm{ml})$ for $24 \mathrm{~h}$. Percentage RFP signal was quantified relative to the non-

558 targeting control siRNA (NTC). ${ }^{*} p<=0.05,{ }^{* *} p<=0.01,{ }^{* * *} p<=0.001$ and ${ }^{* * * *} p<=0.0001$.

559 (C) Microscopy imaging. Representative RFP microscopy images of Fig 5B. (D)

560 Quantification of viral gene expression. Five pmol of siC, siSF3B2 and siSNW1 were

561 transfected in iSLK.r219 cells for $48 \mathrm{~h}$ followed by reactivation with Dox (1 $\mu \mathrm{g} / \mathrm{ml})$ for 24

562 h. PAN RNA, ORF6 and LANA gene expression was quantified using real-time PCR.

$563{ }^{* * *} p<=0.001$ and ${ }^{* * * *} p<=0.0001$. (E) Quantification of progeny virus. Five pmol of siC,

564 siSF3B2 and siSNW1 were transfected in iSLK.r219 cells for $48 \mathrm{~h}$ followed by

565 reactivation with Dox $(1 \mu \mathrm{g} / \mathrm{ml})$ for $24 \mathrm{~h}$. Viral copy number was quantified from tissue 566 culture supernatant using real-time PCR. ${ }^{* * *} \mathrm{p}<=0.001$ and ${ }^{* * * *} \mathrm{p}<=0.0001$.

568 Figure 6. Splicing factor 3B (SF3B1) and SNW1 are suppressors for IFNB1

569 transcription. Five pmol of siC, siSF3B1 or siSNW1 was transfected into 293FT cells

570 for $48 \mathrm{~h}$, followed by polyl:C transfection. Twenty-four post transfection of poly:C, total

571 RNA was harvested and measured IFN-related (a) IFN 31 , (b) IFN 1 and (c) DDX58, or 572 non-related (d) DDX23 mRNAs. ${ }^{* * *} p<=0.001$ and ${ }^{* * *} p<=0.0001$ 


\section{References}

575 1. Chang Y, Cesarman E, Pessin MS, Lee F, Culpepper J, Knowles DM, Moore PS.

576 1994. Identification of herpesvirus-like DNA sequences in AIDS-associated Kaposi's

2. Mesri EA, Cesarman E, Boshoff C. 2010. Kaposi's sarcoma and its associated herpesvirus. Nat Rev Cancer 10:707-719.

580

3. Schulz TF. 2006. The pleiotropic effects of Kaposi's sarcoma herpesvirus. J Pathol

582 208:187-198.

4. Wong EL, Damania B. 2005. Linking KSHV to human cancer. Curr Oncol Rep 7:349356.

5. Cesarman E, Moore PS, Rao PH, Inghirami G, Knowles DM, Chang Y. 1995. In

6. Cesarman E, Knowles DM. 1999. The role of Kaposi's sarcoma-associated herpesvirus vitro establishment and characterization of two acquired immunodeficiency syndromerelated lymphoma cell lines (BC-1 and BC-2) containing Kaposi's sarcoma-associated herpesvirus-like (KSHV) DNA sequences. Blood 86:2708-2714.

(KSHV/HHV-8) in lymphoproliferative diseases. Semin Cancer Biol 9:165-174.

7. Broussard G, Damania B. 2019. KSHV: Immune Modulation and Immunotherapy. Front Immunol 10:3084.

8. Kerur N, Veettil MV, Sharma-Walia N, Bottero V, Sadagopan S, Otageri P, Chandran B. 2011. IFI16 acts as a nuclear pathogen sensor to induce the inflammasome in response to Kaposi Sarcoma-associated herpesvirus infection. Cell Host Microbe 9:363-375.

9. Singh VV, Kerur N, Bottero V, Dutta S, Chakraborty S, Ansari MA, Paudel N, Chikoti L, Chandran B. 2013. Kaposi's sarcoma-associated herpesvirus latency in endothelial and B cells activates gamma interferon-inducible protein 16-mediated inflammasomes. J Virol 87:4417-4431.

10. Inn KS, Lee SH, Rathbun JY, Wong LY, Toth Z, Machida K, Ou JH, Jung JU. 2011. Inhibition of RIG-I-mediated signaling by Kaposi's sarcoma-associated herpesvirusencoded deubiquitinase ORF64. J Virol 85:10899-10904.

11. Zhang Y, Dittmer DP, Mieczkowski PA, Host KM, Fusco WG, Duncan JA, Damania B. 2018. RIG-I Detects Kaposi's Sarcoma-Associated Herpesvirus Transcripts in a RNA Polymerase III-Independent Manner. mBio 9.

12. Zhao Y, Ye X, Dunker W, Song Y, Karijolich J. 2018. RIG-I like receptor sensing of host RNAs facilitates the cell-intrinsic immune response to KSHV infection. Nat Commun 9:4841.

13. West JA, Gregory SM, Sivaraman V, Su L, Damania B. 2011. Activation of plasmacytoid dendritic cells by Kaposi's sarcoma-associated herpesvirus. J Virol 85:895904.

14. West J, Damania B. 2008. Upregulation of the TLR3 pathway by Kaposi's sarcomaassociated herpesvirus during primary infection. J Virol 82:5440-5449.

15. Lagos D, Vart RJ, Gratrix F, Westrop SJ, Emuss V, Wong PP, Robey R, Imami N, Bower M, Gotch F, Boshoff C. 2008. Toll-like receptor 4 mediates innate immunity to Kaposi sarcoma herpesvirus. Cell Host Microbe 4:470-483. 
617 16. Gregory SM, Davis BK, West JA, Taxman DJ, Matsuzawa S, Reed JC, Ting JP,

618 Damania B. 2011. Discovery of a viral NLR homolog that inhibits the inflammasome.

$619 \quad$ Science 331:330-334.

620 17. Myoung J, Lee SA, Lee HR. 2019. Beyond Viral Interferon Regulatory Factors:

621

622

623

624

625

626

627

628

18. Lee HR, Kim MH, Lee JS, Liang C, Jung JU. 2009. Viral interferon regulatory factors. J Interferon Cytokine Res 29:621-627.

19. Takaoka A, Tamura T, Taniguchi T. 2008. Interferon regulatory factor family of transcription factors and regulation of oncogenesis. Cancer Sci 99:467-478.

20. Jacobs SR, Damania B. 2011. The viral interferon regulatory factors of KSHV: immunosuppressors or oncogenes? Front Immunol 2:19.

21. Burysek L, Yeow WS, Lubyova B, Kellum M, Schafer SL, Huang YQ, Pitha PM. 1999. Functional analysis of human herpesvirus 8-encoded viral interferon regulatory factor 1 and its association with cellular interferon regulatory factors and p300. J Virol 73:7334-7342.

22. Li M, Damania B, Alvarez X, Ogryzko V, Ozato K, Jung JU. 2000. Inhibition of p300 histone acetyltransferase by viral interferon regulatory factor. Mol Cell Biol 20:82548263.

23. Xiang Q, Ju H, Li Q, Mei SC, Chen D, Choi YB, Nicholas J. 2018. Human Herpesvirus 8 Interferon Regulatory Factors 1 and 3 Mediate Replication and Latency Activities via Interactions with USP7 Deubiquitinase. J Virol 92.

24. Koch S, Damas M, Freise A, Hage E, Dhingra A, Ruckert J, Gallo A, Kremmer E, Tegge W, Bronstrup M, Brune W, Schulz TF. 2019. Kaposi's sarcoma-associated herpesvirus vIRF2 protein utilizes an IFN-dependent pathway to regulate viral early gene expression. PLoS Pathog 15:e1007743.

25. Yu HR, Kim YJ, Lee HR. 2018. KSHV vIRF4 enhances BCL6 transcription via downregulation of IRF4 expression. Biochem Biophys Res Commun 496:1128-1133.

26. Hwang SW, Kim D, Jung JU, Lee HR. 2017. KSHV-encoded viral interferon regulatory factor 4 (vIRF4) interacts with IRF7 and inhibits interferon alpha production. Biochem Biophys Res Commun 486:700-705.

27. Roux KJ, Kim DI, Raida M, Burke B. 2012. A promiscuous biotin ligase fusion protein identifies proximal and interacting proteins in mammalian cells. J Cell Biol 196:801-810.

28. Branon TC, Bosch JA, Sanchez AD, Udeshi ND, Svinkina T, Carr SA, Feldman JL, Perrimon N, Ting AY. 2018. Efficient proximity labeling in living cells and organisms with TurboID. Nat Biotechnol 36:880-887.

29. Motani K, Kosako H. 2020. BioID screening of biotinylation sites using the avidin-like protein Tamavidin 2-REV identifies global interactors of stimulator of interferon genes (STING). J Biol Chem 295:11174-11183.

30. Bozal-Basterra L, Gonzalez-Santamarta M, Muratore V, Bermejo-Arteagabeitia A, Da Fonseca C, Barroso-Gomila O, Azkargorta M, Iloro I, Pampliega O, Andrade R, Martin-Martin N, Branon TC, Ting AY, Rodriguez JA, Carracedo A, Elortza F, Sutherland JD, Barrio R. 2020. LUZP1, a novel regulator of primary cilia and the actin cytoskeleton, is a contributing factor in Townes-Brocks Syndrome. Elife 9.

661

31. Cho KF, Branon TC, Rajeev S, Svinkina T, Udeshi ND, Thoudam T, Kwak C, Rhee 
32. May DG, Scott KL, Campos AR, Roux KJ. 2020. Comparative Application of BioID and TurboID for Protein-Proximity Biotinylation. Cells 9.

33. Shinoda N, Hanawa N, Chihara T, Koto A, Miura M. 2019. Dronc-independent basal executioner caspase activity sustains Drosophila imaginal tissue growth. Proc Natl Acad Sci U S A 116:20539-20544.

34. Mair A, Xu SL, Branon TC, Ting AY, Bergmann DC. 2019. Proximity labeling of protein complexes and cell-type-specific organellar proteomes in Arabidopsis enabled by TurboID. Elife 8.

35. Zhang Y, Song G, Lal NK, Nagalakshmi U, Li Y, Zheng W, Huang PJ, Branon TC, Ting AY, Walley JW, Dinesh-Kumar SP. 2019. TurboID-based proximity labeling reveals that UBR7 is a regulator of N NLR immune receptor-mediated immunity. Nat Commun 10:3252.

36. Arora D, Abel NB, Liu C, Van Damme P, Yperman K, Eeckhout D, Vu LD, Wang J, Tornkvist A, Impens F, Korbei B, Van Leene J, Goossens A, De Jaeger G, Ott T, Moschou PN, Van Damme D. 2020. Establishment of Proximity-dependent Biotinylation Approaches in Different Plant Model Systems. Plant Cell doi:10.1105/tpc.20.00235.

37. Zhang Y, Li Y, Yang X, Wen Z, Nagalakshmi U, Dinesh-Kumar SP. 2020. TurboIDBased Proximity Labeling for In Planta Identification of Protein-Protein Interaction Networks. J Vis Exp doi:10.3791/60728.

38. Larochelle M, Bergeron D, Arcand B, Bachand F. 2019. Proximity-dependent biotinylation mediated by TurboID to identify protein-protein interaction networks in yeast. J Cell Sci 132.

39. Izumiya Y, Izumiya C, Van Geelen A, Wang DH, Lam KS, Luciw PA, Kung HJ. 2007. Kaposi's sarcoma-associated herpesvirus-encoded protein kinase and its interaction with K-bZIP. J Virol 81:1072-1082.

40. Tischer BK, Smith GA, Osterrieder N. 2010. En passant mutagenesis: a two step markerless red recombination system. Methods Mol Biol 634:421-430.

41. Brulois KF, Chang H, Lee AS, Ensser A, Wong LY, Toth Z, Lee SH, Lee HR, Myoung J, Ganem D, Oh TK, Kim JF, Gao SJ, Jung JU. 2012. Construction and manipulation of a new Kaposi's sarcoma-associated herpesvirus bacterial artificial chromosome clone. J Virol 86:9708-9720.

42. Izumiya Y, Izumiya C, Hsia D, Ellison TJ, Luciw PA, Kung HJ. 2009. NF-kappaB serves as a cellular sensor of Kaposi's sarcoma-associated herpesvirus latency and negatively regulates K-Rta by antagonizing the RBP-Jkappa coactivator. J Virol 83:44354446.

43. Fakhari FD, Dittmer DP. 2002. Charting latency transcripts in Kaposi's sarcomaassociated herpesvirus by whole-genome real-time quantitative PCR. J Virol 76:62136223.

44. Nesvizhskii AI, Keller A, Kolker E, Aebersold R. 2003. A statistical model for identifying proteins by tandem mass spectrometry. Anal Chem 75:4646-4658.

45. Zhou Y, Zhou B, Pache L, Chang M, Khodabakhshi AH, Tanaseichuk O, Benner C, Chanda SK. 2019. Metascape provides a biologist-oriented resource for the analysis of systems-level datasets. Nat Commun 10:1523.

46. Goodacre N, Devkota P, Bae E, Wuchty S, Uetz P. 2020. Protein-protein interactions of human viruses. Semin Cell Dev Biol 99:31-39. 
47. Nakajima KI, Guevara-Plunkett S, Chuang F, Wang KH, Lyu Y, Kumar A, Luxardi G, Izumiya C, Soulika A, Campbell M, Izumiya Y. 2020. Rainbow Kaposi's Sarcoma-Associated Herpesvirus Revealed Heterogenic Replication with Dynamic Gene Expression. J Virol 94.

48. Seo T, Park J, Lee D, Hwang SG, Choe J. 2001. Viral interferon regulatory factor 1 of Kaposi's sarcoma-associated herpesvirus binds to p53 and represses p53-dependent transcription and apoptosis. J Virol 75:6193-6198.

49. Shin YC, Nakamura H, Liang X, Feng P, Chang H, Kowalik TF, Jung JU. 2006. Inhibition of the ATM/p53 signal transduction pathway by Kaposi's sarcoma-associated herpesvirus interferon regulatory factor 1. J Virol 80:2257-2266.

50. Sun C. 2020. The SF3b complex: splicing and beyond. Cell Mol Life Sci 77:3583-3595.

51. Malcovati L, Papaemmanuil E, Bowen DT, Boultwood J, Della Porta MG, Pascutto C, Travaglino E, Groves MJ, Godfrey AL, Ambaglio I, Galli A, Da Via MC, Conte S, Tauro S, Keenan N, Hyslop A, Hinton J, Mudie LJ, Wainscoat JS, Futreal PA, Stratton MR, Campbell PJ, Hellstrom-Lindberg E, Cazzola M, Chronic Myeloid Disorders Working Group of the International Cancer Genome C, of the Associazione Italiana per la Ricerca sul Cancro Gruppo Italiano Malattie M. 2011. Clinical significance of SF3B1 mutations in myelodysplastic syndromes and myelodysplastic/myeloproliferative neoplasms. Blood 118:6239-6246.

52. Dolatshad H, Pellagatti A, Liberante FG, Llorian M, Repapi E, Steeples V, Roy S, Scifo L, Armstrong RN, Shaw J, Yip BH, Killick S, Kusec R, Taylor S, Mills KI, Savage KI, Smith CWJ, Boultwood J. 2016. Cryptic splicing events in the iron transporter ABCB7 and other key target genes in SF3B1-mutant myelodysplastic syndromes. Leukemia 30:2322-2331.

53. Papaemmanuil E, Cazzola M, Boultwood J, Malcovati L, Vyas P, Bowen D, Pellagatti A, Wainscoat JS, Hellstrom-Lindberg E, Gambacorti-Passerini C, Godfrey AL, Rapado I, Cvejic A, Rance R, McGee C, Ellis P, Mudie LJ, Stephens PJ, McLaren S, Massie CE, Tarpey PS, Varela I, Nik-Zainal S, Davies HR, Shlien A, Jones D, Raine K, Hinton J, Butler AP, Teague JW, Baxter EJ, Score J, Galli A, Della Porta MG, Travaglino E, Groves M, Tauro S, Munshi NC, Anderson KC, ElNaggar A, Fischer A, Mustonen V, Warren AJ, Cross NCP, Green AR, Futreal PA, Stratton MR, Campbell PJ, Consortium ICG. 2011. Somatic SF3B1 Mutation in Myelodysplasia with Ring Sideroblasts. New England Journal of Medicine 365:13841395.

54. Singh S, Ahmed D, Dolatshad H, Tatwavedi D, Schulze U, Sanchi A, Ryley S, Dhir A, Carpenter L, Watt SM, Roberts DJ, Abdel-Aal AM, Sayed SK, Mohamed SA, Schuh A, Vyas P, Killick S, Kotini AG, Papapetrou EP, Wiseman DH, Pellagatti A, Boultwood J. 2020. SF3B1 mutations induce R-loop accumulation and DNA damage in MDS and leukemia cells with therapeutic implications. Leukemia 34:2525-2530.

55. De Arras L, Alper S. 2013. Limiting of the innate immune response by SF3A-dependent control of MyD88 alternative mRNA splicing. PLoS Genet 9:e1003855.

56. Zhang Q, Liang T, Gu S, Ye Y, Liu S. 2020. SNW1 interacts with IKKgamma to positively regulate antiviral innate immune responses against influenza A virus infection. Microbes Infect doi:10.1016/j.micinf.2020.07.009.

57. Zhang H, Ni G, Damania B. 2020. ADAR1 Facilitates KSHV Lytic Reactivation by Modulating the RLR-Dependent Signaling Pathway. Cell Rep 31:107564. 
bioRxiv preprint doi: https://doi.org/10.1101/2020.10.16.343483; this version posted October 17, 2020. The copyright holder for this preprint (which was not certified by peer review) is the author/funder, who has granted bioRxiv a license to display the preprint in perpetuity. It is made available under aCC-BY-NC-ND 4.0 International license.

755

756

757

758

58. Gupta S, Yla-Anttila P, Sandalova T, Achour A, Masucci MG. 2020. Interaction With 14-3-3 Correlates With Inactivation of the RIG-I Signalosome by Herpesvirus Ubiquitin Deconjugases. Front Immunol 11:437. 
760 Table 1. Primers, Plasmid, and Gene block DNA sequence used for BAC16 recombination $761 \quad\left(5^{\prime}->3 '\right)$

\begin{tabular}{|c|c|}
\hline $\begin{array}{l}\text { Template: pEPkan-S } \\
\text { Addgene (Plasmid } \\
\text { \#41017) }\end{array}$ & \\
\hline $\begin{array}{l}\text { mini-TurboID-Kan } \\
\text { SalI-S }\end{array}$ & $\begin{array}{l}\text { GCCCGTCGTCGACTCCACCAATCAGTACCTCTTGGATCGGATTGGGGAGTTGAAG } \\
\text { AGCGGTAGGGATAACAGGGTAATCGATTT }\end{array}$ \\
\hline Kan SalI-AS & AAAGTCGACGCCAGTGTTACAACCAATTAACC \\
\hline $\begin{array}{l}\text { Flagx3 mini-TurboID } \\
\text { codon optimized } \\
\text { fragment }(\mathrm{KpnI} / \mathrm{SacII}) \\
\text { cloned into } \mathrm{pBS} \mathrm{SK}+ \\
\text { vector with Gibson } \\
\text { assembly }\end{array}$ & $\begin{array}{l}\text { ATGGATTATAAGGATGATGACAAGGGGGACTATAAAGACGACGATAAAGGCGAC } \\
\text { TATAAGGACGATGATAAAGCGTCCATACCGCTGCTGAATGCAAAACAGATCCTGG } \\
\text { GGCAGTTGGATGGTGGAAGCGTCGCAGTGCTGCCCGTCGTCGACTCCACCAATCA } \\
\text { GTACCTCTTGGATCGGATTGGGGAGTTGAAGAGCGGTGATGCGTGCATCGCGGA } \\
\text { GTACCAGCAAGCAGGCAGAGGTAGCCGCGGACGAAAATGTTTAGTCCTTTGGT } \\
\text { GCGAACCTGTACCTCAGCATGTTCTGGAGGCTCAAGAGAGGCCCCGCGGCGATTG } \\
\text { GACTTGGCCCAGTAATCGGGATCGTCATGGCTGAGGCGCTCAGAAAACTCGGAGC } \\
\text { TGATAAGGTTAGAGTAAAATGGCCGAACGACCTTTATTTGCAAGACCGAAAATTG } \\
\text { GCTGGGATATTGGTGGAACTTGCGGGCATTACCGGCGACGCGGCACAAATCGTCA } \\
\text { TAGGTGCCGGTATTAATGTGGCAATGCGCCGCGTTGAAGAGAGCGTGGTAAATCA } \\
\text { GGGATGGATAACCCTGCAAGAGGCAGGAATCAACCTGGACCGCAACACCCTGGCT } \\
\text { GCTATGCTCATTCGGGAACTGAGAGCTGCGTTGGAGCTCTTTGAACAGGAAGGGC } \\
\text { TTGCACCGTACCTCAGTCGATGGGAAAAATTGGATAACTTCATAAATCGGCCTGTG } \\
\text { AAACTCATCATAGGCGACAAGGAAATCTTTGGCATTAGTCGAGGGATTGATAAGC } \\
\text { AAGGCGCACTCTTGCTCGAACAGGACGGAGTTATCAAACCTTGGATGGGTGGCGA } \\
\text { AATTAGTCTCAGAAGTGCAGAGAAGGAGTTTAGCCGAGCGGACTAA }\end{array}$ \\
\hline $\begin{array}{l}\text { Template: pBS-mini- } \\
\text { TurboID Kan }\end{array}$ & \\
\hline $\begin{array}{l}\text { K9 mini-TurboID-S } \\
\text { (vIRF-1) }\end{array}$ & $\begin{array}{l}\text { CTGTCGCCTCTCTATATCTGATGGCCGGTGGCTCCCCGGCATAGCTGTGCTTACCAC } \\
\text { TGGACATTGCGGCGCGAGCTAGTCTGGTTGCGGGACAATGGATTATAAGGATGA } \\
\text { TGACAAGGGGGAC }\end{array}$ \\
\hline K9 mini-TurboID-AS & $\begin{array}{l}\text { GTTCCCGGTGACCCTTGTGACAAACAAGGTTTTTTGGGTATCGCCCCAGGCGCCCC } \\
\text { AAAAGGGTTCGGTCTTTGGCCTGGGTCCATGTCCGCTCGGCTAAACTCCTTCTCTG }\end{array}$ \\
\hline $\begin{array}{l}\text { K10 mini-TurboID-S } \\
\text { (vIRF-4) }\end{array}$ & $\begin{array}{l}\text { TAGCAAGAAGGGGGGCACTATAAGGCTCAGTCGGGACTGTGCCTCAAAGACGAA } \\
\text { CGCCGATCGGTTTCTGTGTCGGACCATGGATTATAAGGATGATGACAAGGGGGA } \\
\text { C }\end{array}$ \\
\hline K10 mini-TurboID-AS & $\begin{array}{l}\text { AAACCAGGAAAAATAGGGAAACTTATTGTTTTCAAGGGCATCAATAATCCATAACG } \\
\text { TGGCCCATTCTGAGCCACCGGCTTTAGGGTCCGCTCGGCTAAACTCCTTCTCTG }\end{array}$ \\
\hline
\end{tabular}

762 Restriction enzyme sites used to clone kanamycin cassette were underlined. Start and stop codon

763 for mini-TurboID coding sequence was marked with bold-underlined. Italic letters indicate

764 homology arms with KSHV genome for recombination. Bold letters anneal to cloned mini-

765 TurboID cassette for amplification DNA fragment for recombination.

766 
(i) Clone miniTurbo tag sequence
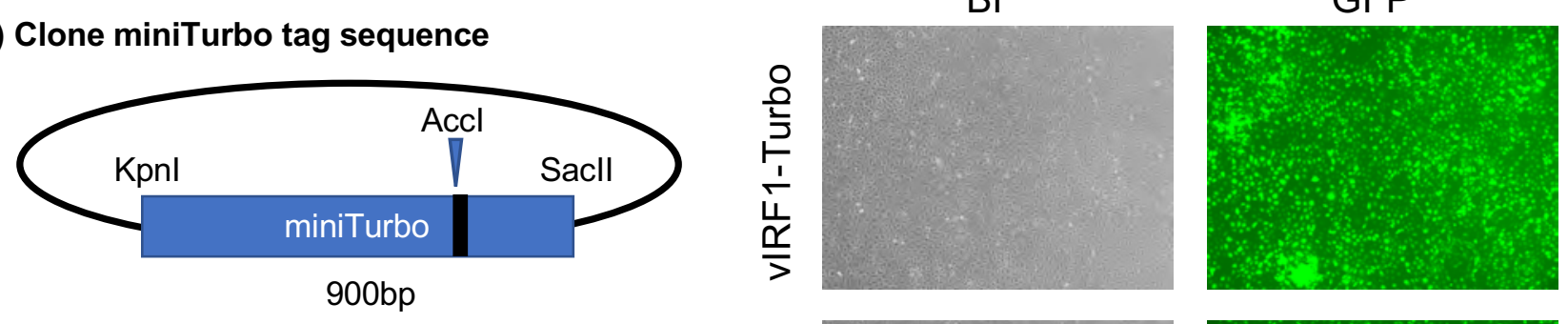

(ii) PCR

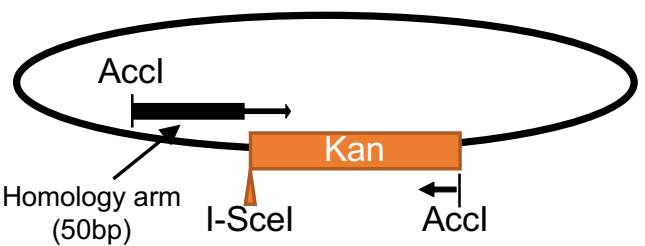

(iii) Ligation
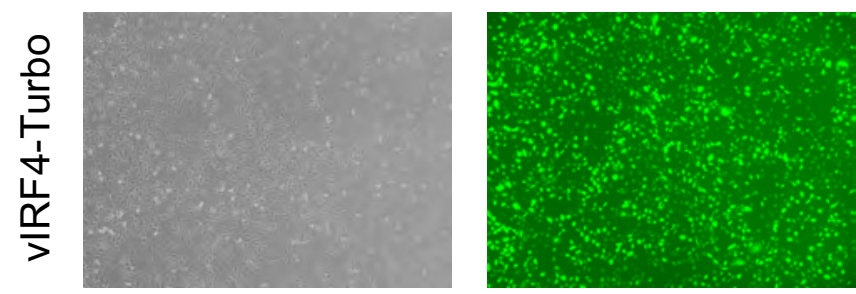

C

Fig. 1

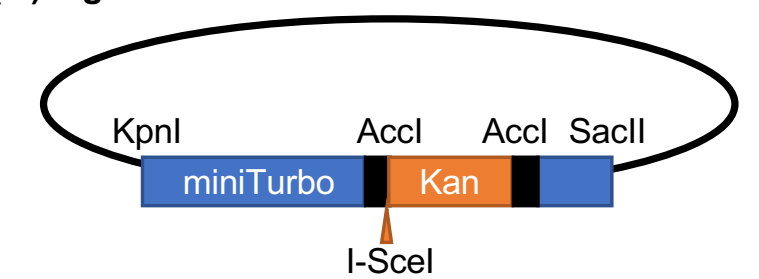

(iv) PCR

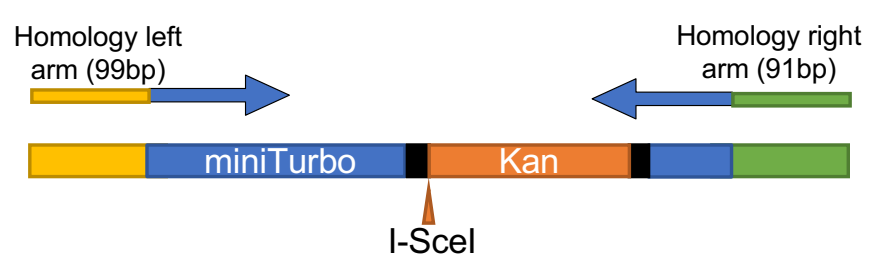

(v) Recombination with BAC
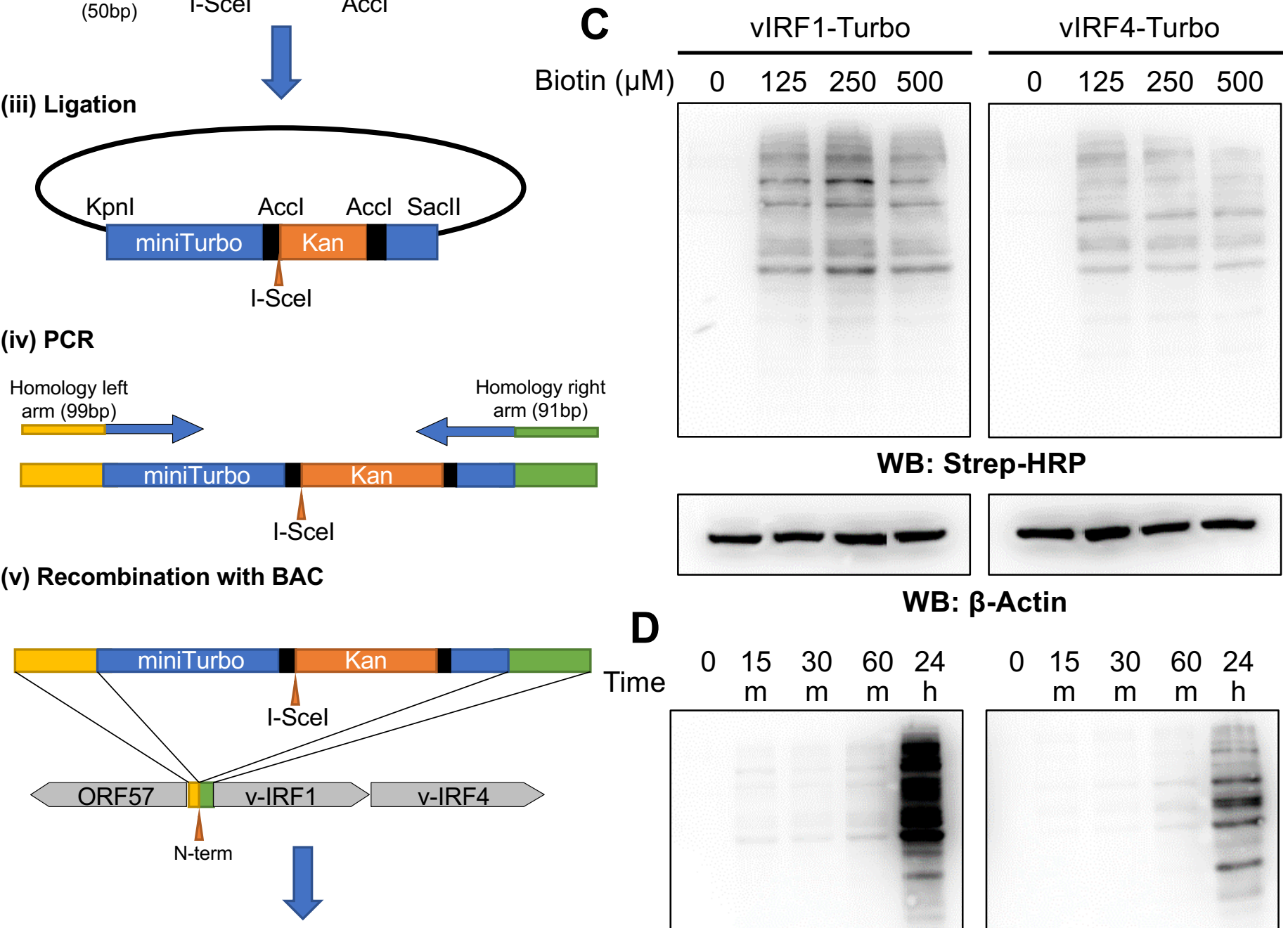

(vi) Kanamycin selection and PCR screening

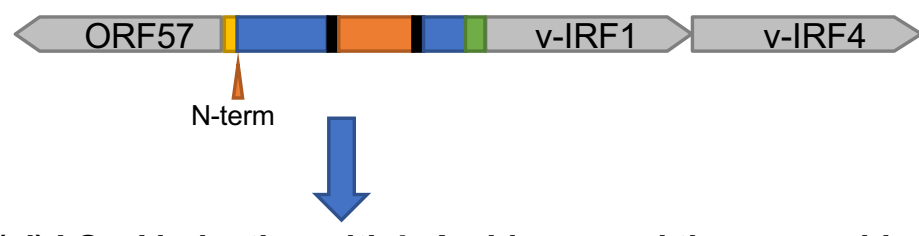

D

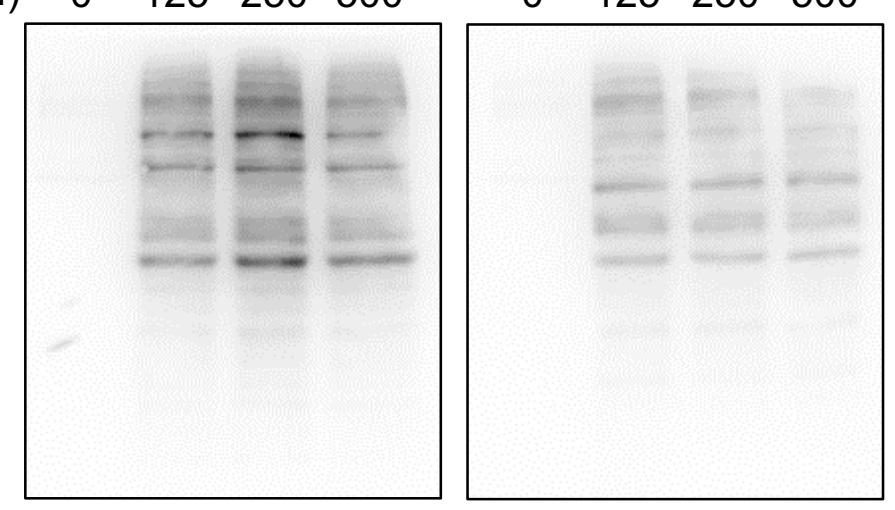

WB: Strep-HRP

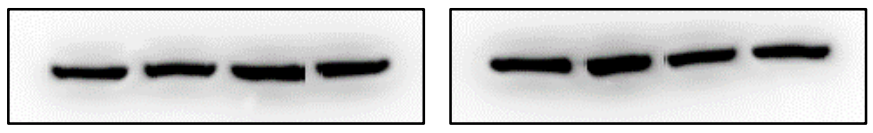

WB: $\beta$-Actin

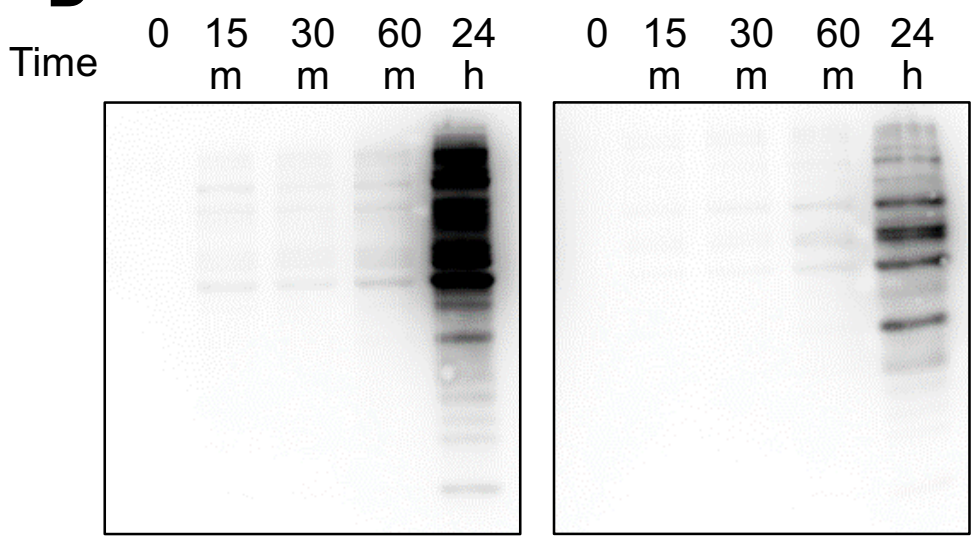

Vi) I-Scel induction with L-Arabinose and then recombination,

WB: Strep-HRP

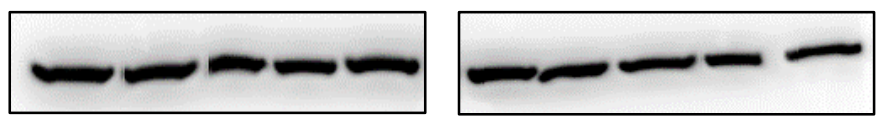

WB: $\beta$-Actin check integrity of BAC by restriction digestion and PCR

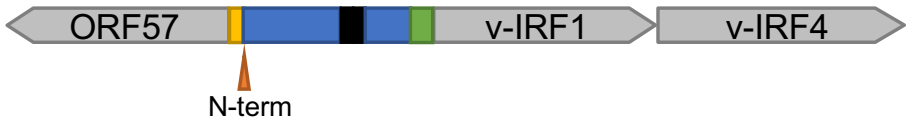


Fig. 2
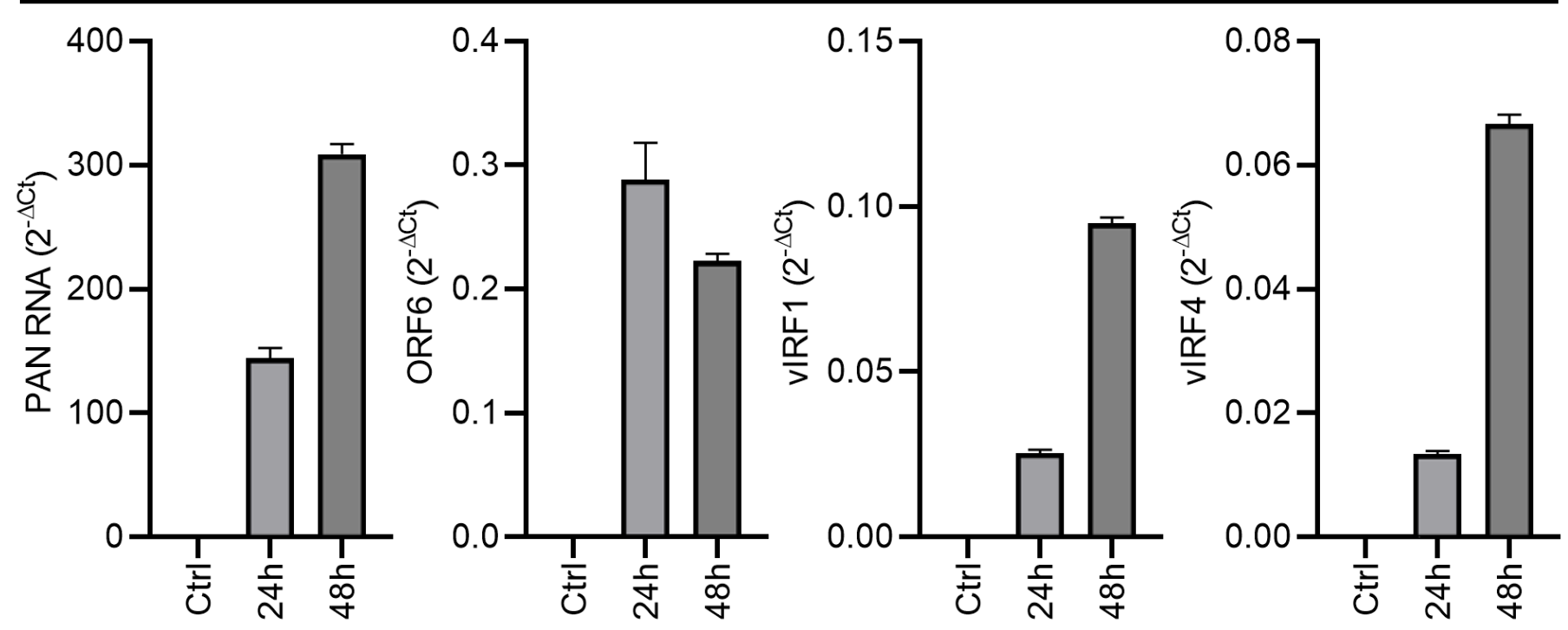

vIRF4-Turbo

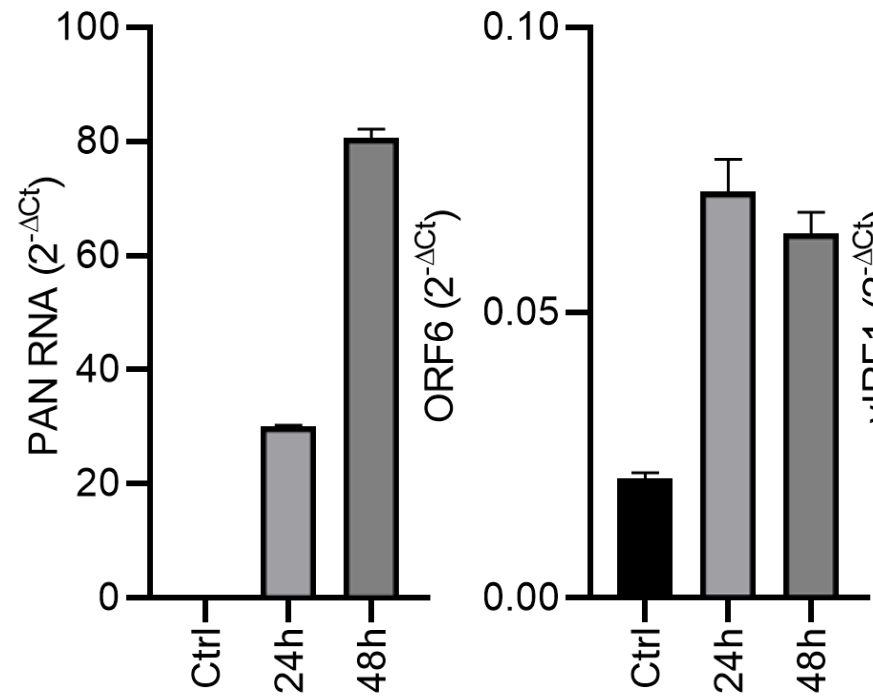

0.020

B

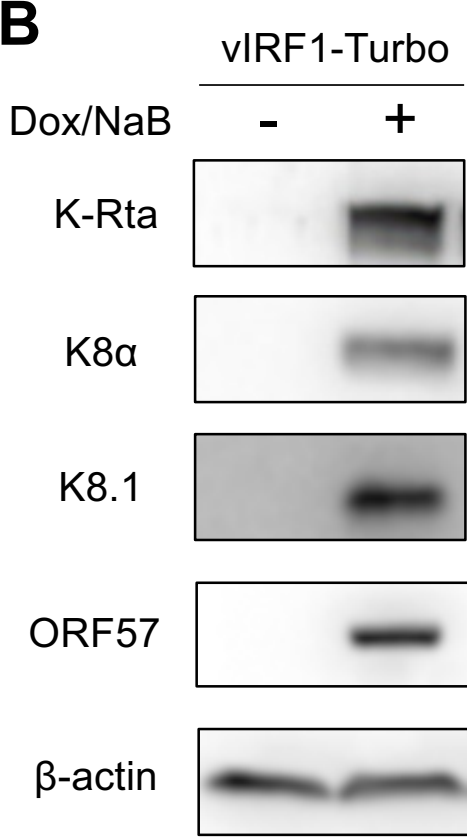

vIRF4-Turbo
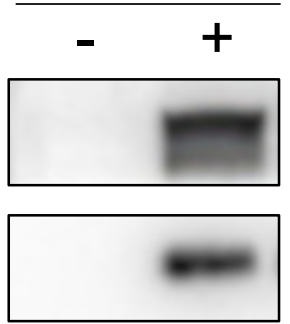

$\stackrel{\sqcup}{\infty}$
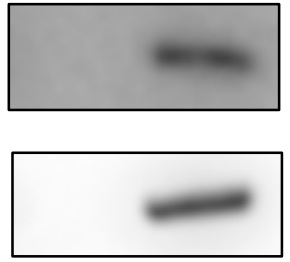

$\frac{1}{0}$

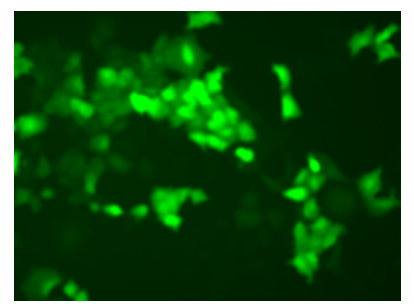

vIRF4-Turbo
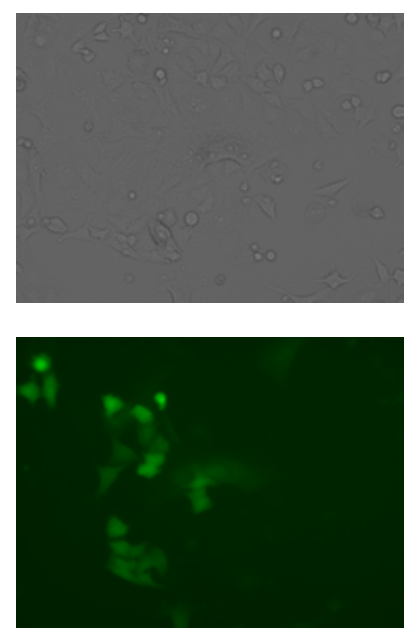


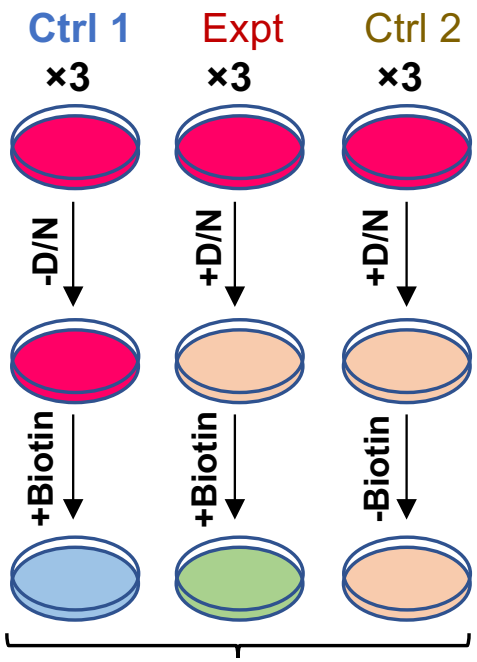

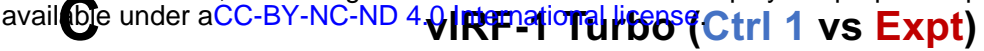

Fig. 3

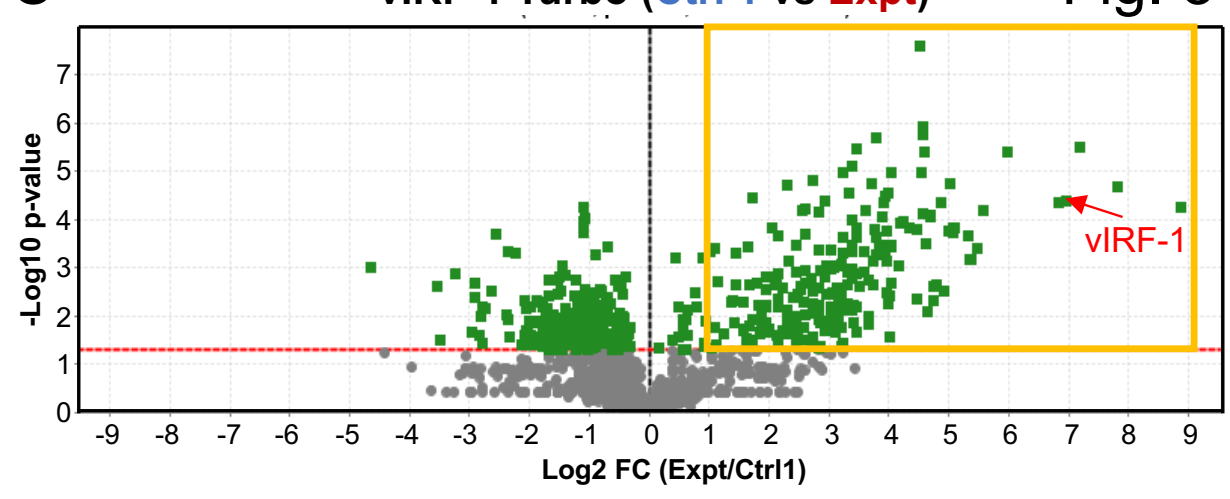

V-IRF-1 Turbo (Ctrl 2 vs Expt)

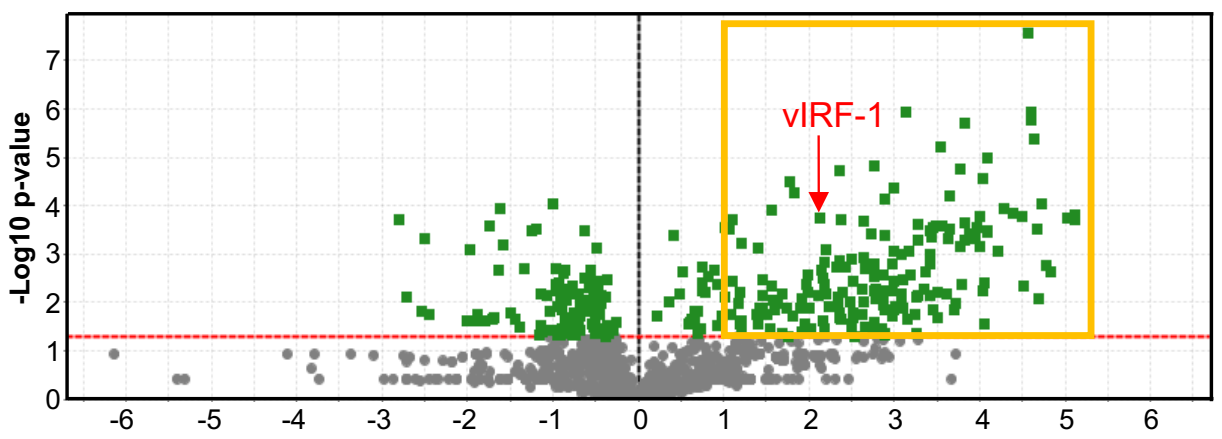

Incubation at $4^{\circ} \mathrm{C}, 1 \mathrm{~h}$

3x Washing ice-cold PBS
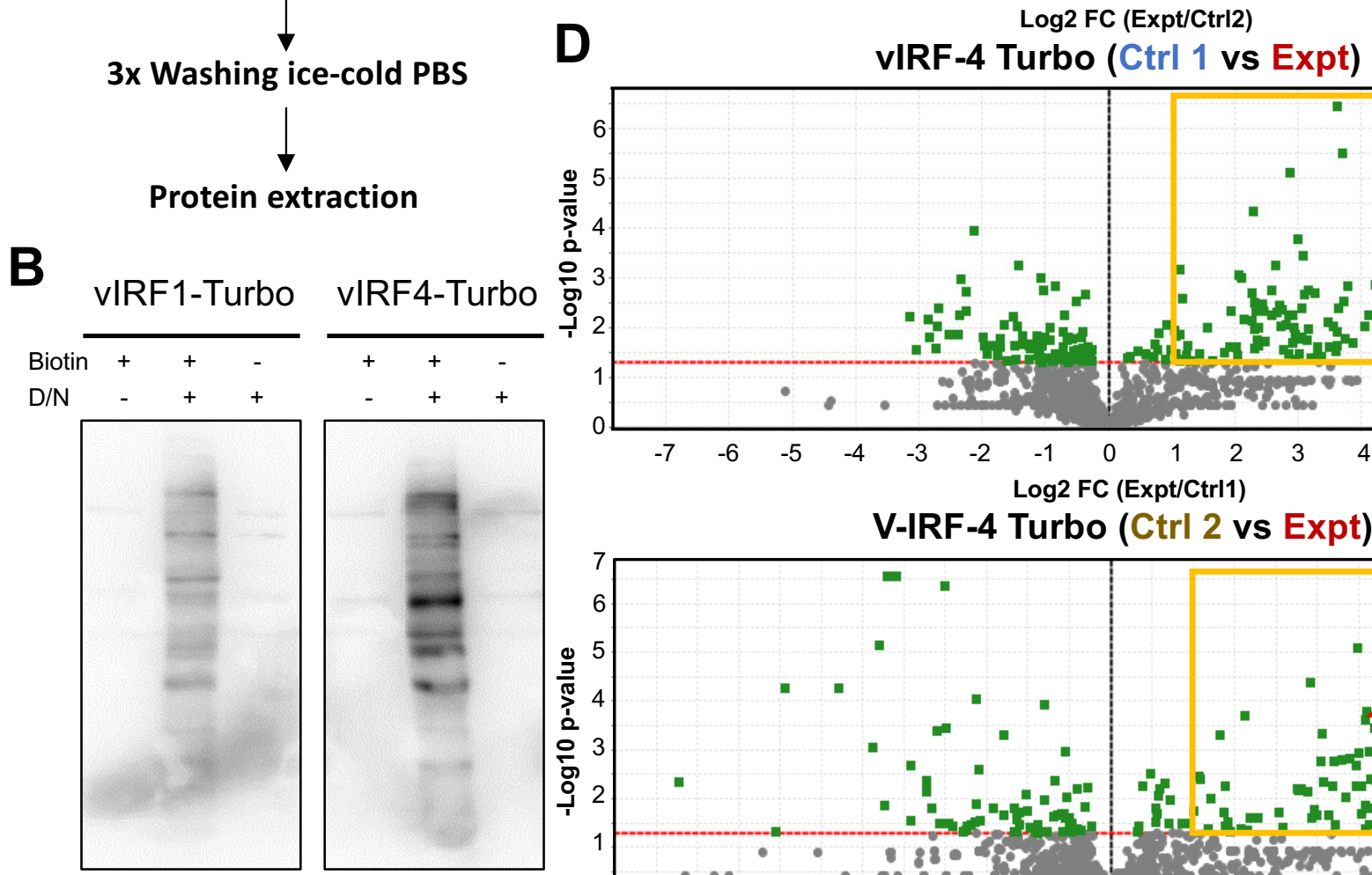

WB: Strep-HRP

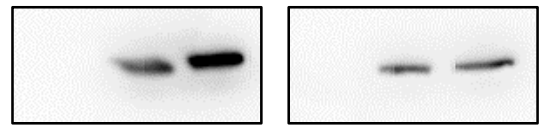

WB: Flag
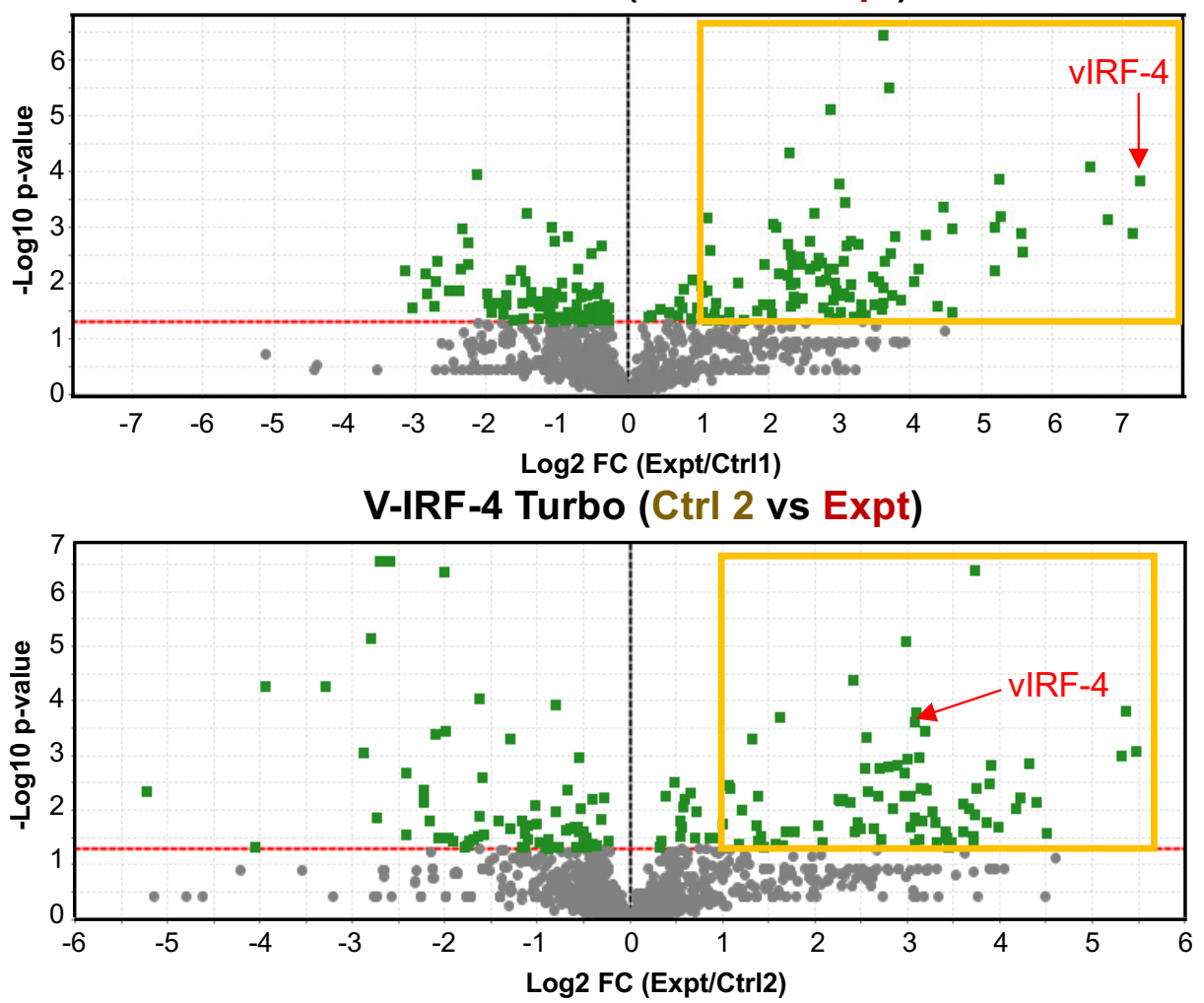

Significant Non-Significant ----. Significance Threshold -.-.. Zero-Fold Change

E $\quad$ VIRF1-Turbo

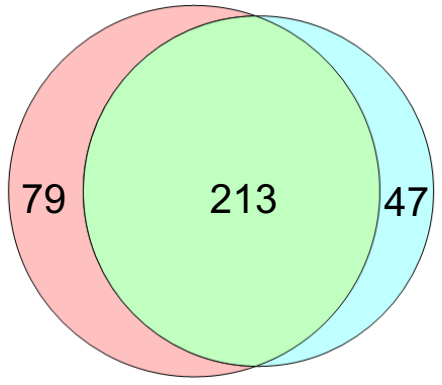

vIRF4-Turbo

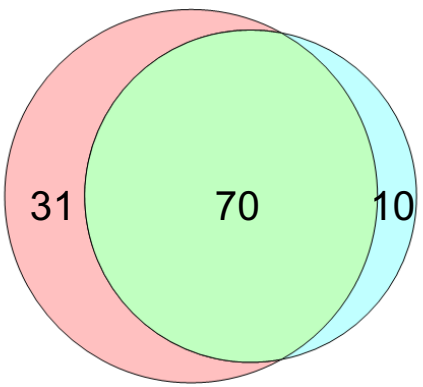


bioRxiv preprint doi: https://doi.org/10.1101/2020.10.16.343483; this version posted October 17, 2020. The copyright holder for this preprint

(which was not certified by peer review) is the author/funder, who has granted bioRxiv a license to display the preprint in perpetuity is made

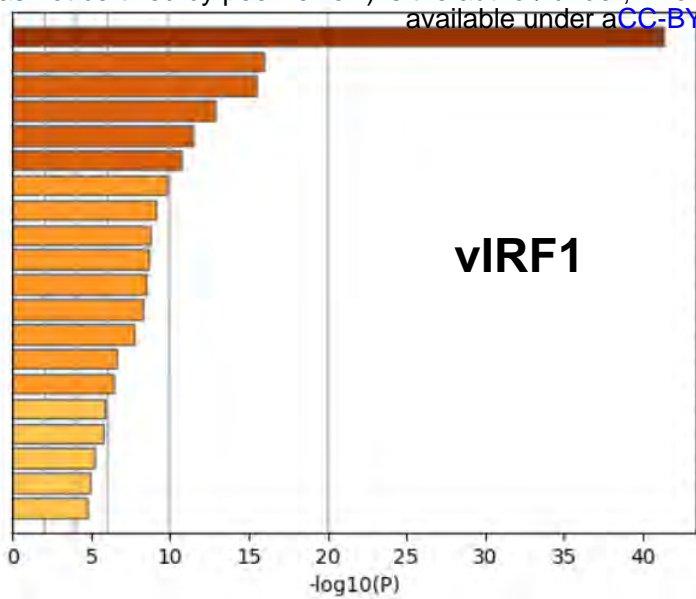

R-HA-37009: ma processing

R-HSA-3700989: Transcriptional Regulation by TP53

GO:0050684: regulation of MRNA processing

R-HSA-112382: Formation of RNA Pol II elongation complex

GO:0032786: positive regulation of DNA-templated transcription, elongation

R-HSA-72165: mRNA Splicing - Minor Pathway

GO:0051255: spindle midzone assembly

GO:0031124: mRNA 3'-end processing

GO:0032204: regulation of telomere maintenance

GO:0022618: ribonucleoprotein complex assembly

GO:0071103: DNA conformation change

M101: PID HDAC CLASSI PATHWAY

GO:0006403: RNA localization

GO:0043254: regulation of protein complex assembly

GO:0006281: DNA repair

GO:0010564: regulation of cell cycle process

GO:0051052: regulation of DNA metabolic process

CORUM:1191: RNA pol II containing coactivator complex Tat-SF

GO:0010639: negative regulation of organelle organization

M14: PID AURORA B PATHWAY

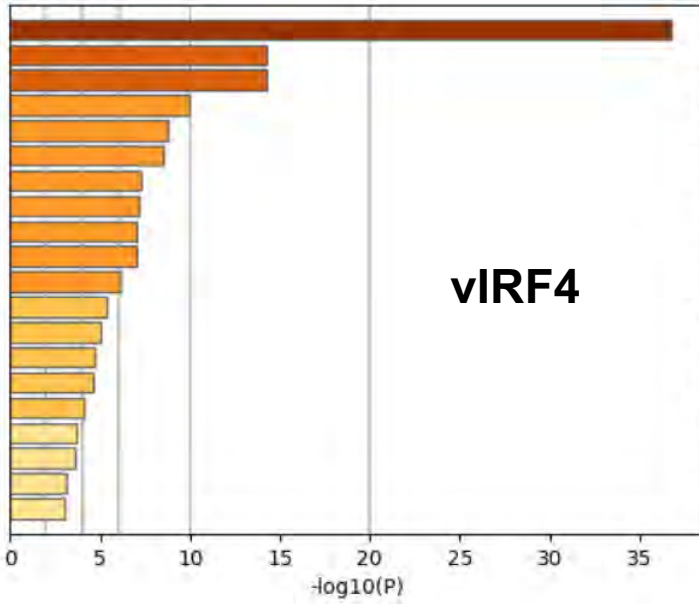

GO:0006397: mRNA processing

GO:0050684: regulation of mRNA processing

GO:0006378: mRNA polyadenylation

R-HSA-72165: mRNA Splicing - Minor Pathway

GO:1900363: regulation of MRNA polyadenylation

GO:0032204: regulation of telomere maintenance

GO:0006913: nucleocytoplasmic transport

R-HSA-3700989: Transcriptional Regulation by TP53

CORUM:3064: RNA polymerase II complex, chromatin structure modifying

GO:0050434: positive regulation of viral transcription

GO:0072331: signal transduction by $\mathrm{p} 53$ class mediator

GO:0022618: ribonucleoprotein complex assembly

M236: PID DELTA NP63 PATHWAY

GO:0032392: DNA geometric change

R-HSA-2028269: Signaling by Hippo

GO:1903313: positive regulation of mRNA metabolic process

GO:0006367: transcription initiation from RNA polymerase II promoter

GO:0009895: negative regulation of catabolic process

GO:0045815: positive regulation of gene expression, epigenetic

GO:0031668: cellular response to extracellular stimulus

B
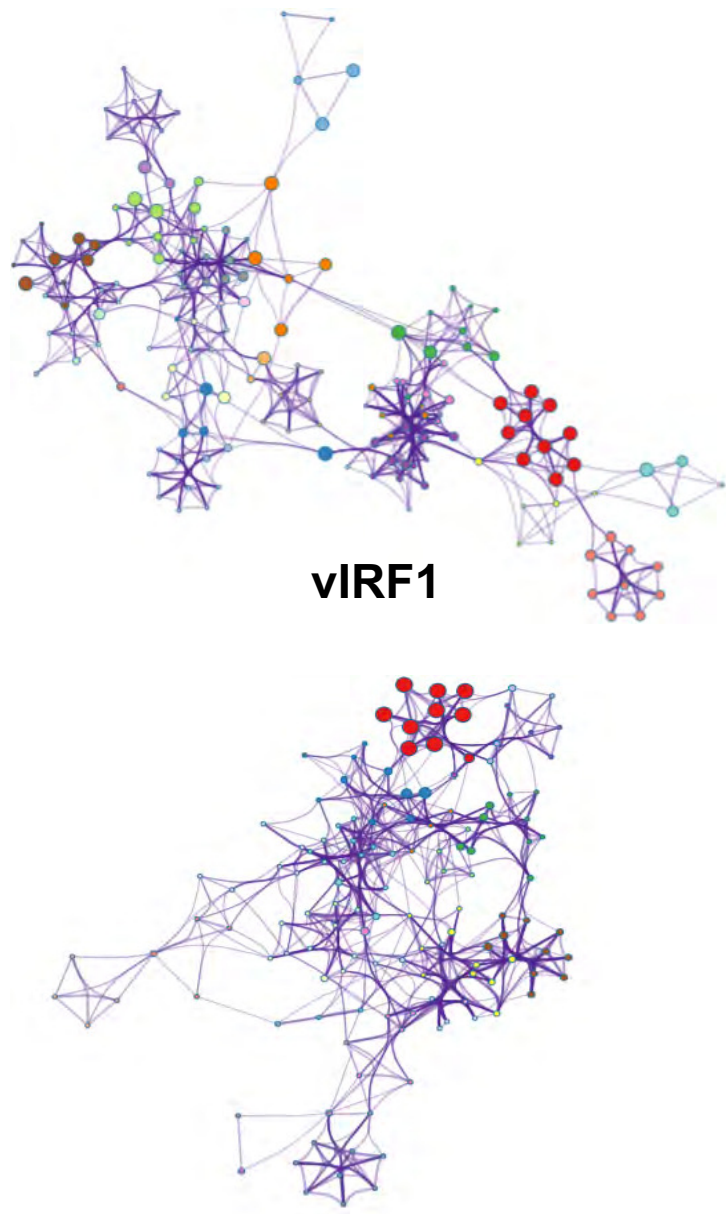

vIRF4
mRNA processing

Transcriptional Regulation by TP53

regulation of mRNA processing

Formation of RNA Pol II elongation complex

positive regulation of DNA-templated transcription. mRNA Splicing - Minor Pathway

spindle midzone assembly

IIRNA 3 '-end processing

Elegulation of telomere maintenance

aribonucleoprotein complex assembly

DNA conformation change

PID HDAC CLASSI PATHWAY

RNA localization

regulation of protein complex assembly

DNA repair

regulation of cell cycle process

regulation of DNA metabolic process

RNA pol II containing coactivator complex Tat-SF

- negative regulation of organelle organization

PID AURORA B PATHWAY

mRNA processing

regulation of mRNA processing

a mRNA polyadenylation

ImRNA Splicing - Minor Pathway

regulation of mRNA polyadenylation

regulation of telomere maintenance

nucleocytoplasmic transport

- Transcriptional Regulation by TP53

RNA polymerase II complex, chromatin structure mod

- positive regulation of viral transcription

signal transduction by $p 53$ class mediator

ribonucleoprotein complex assembly

PID DELTA NP63 PATHWAY

DNA geometric change

Signaling by Hippo

positive regulation of mRNA metabolic process

transcription initiation from RNA polymerase II promo

negative regulation of catabolic process

positive regulation of gene expression, epigenetic

celluiar response to extracellular stimulus 
bioRxiv preprint doi: https://doi.org/10.1101/2020.10.16.343483; this version posted October 17, 2020. The copyright holder for this preprint (which was not certified by peer review) is the author/funder, who has granted bioRxiv a license to display the preprint in perpetuity $\mathrm{J}$ is made A vIRF-1 vs vIRF-4 available under aCC-BY-NC-ND 4.0 International license.

Fig. 5

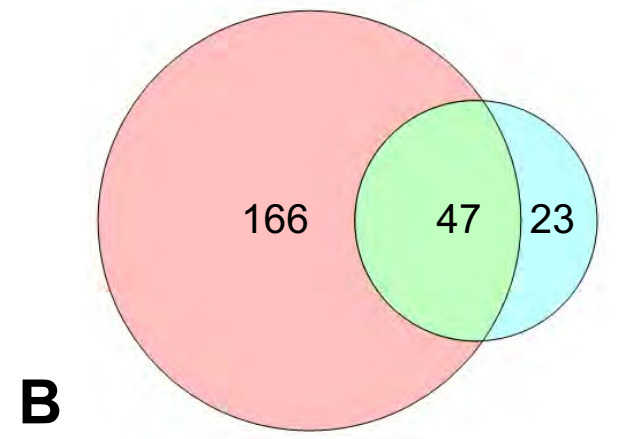

\section{Cellular Proteins}

\begin{tabular}{lllll}
\hline IFI16 & HNRNPA1 & AGFG1 & ADNP & LASP1 \\
HELLS & SYMPK & GTF2F2 & UBAP2L & SNRPD1 \\
PRPF3 & GTF2F1 & ZC3H14 & SNW1 & CHD4 \\
YAP1 & ZNF217 & USP7 & GATAD2B & RBM27 \\
XAB2 & DDX42 & FOSL2 & TJP2 & RBM22 \\
MYO6 & SF3B2 & ZNF638 & CCNT1 & SF3B1 \\
MRE11A & YLPM1 & SF3B3 & RBM10 & SF1 \\
SUGP2 & HN1 & SUPT5H & PCNP & HNRNPA2B1 \\
TPX2 & ZNF281 & UPF1 & HN1L &
\end{tabular}

Viral Proteins

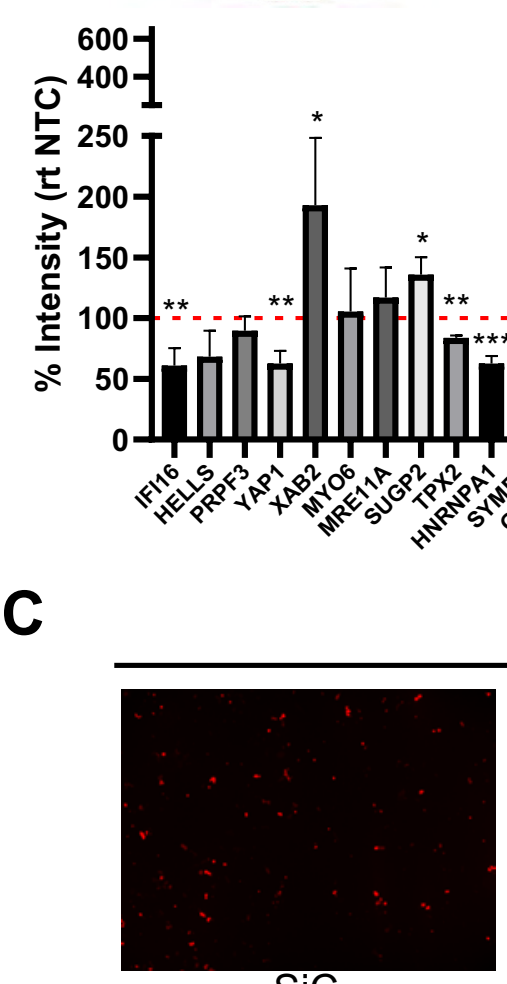

Knockdown regulates KSHV reactivation

SiRNA

$\mathrm{SiC}$

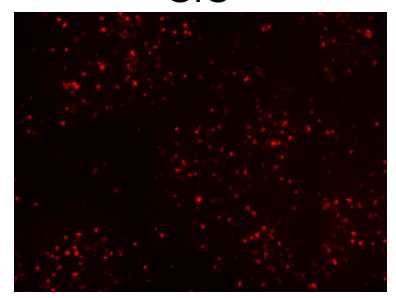

siSF3B2

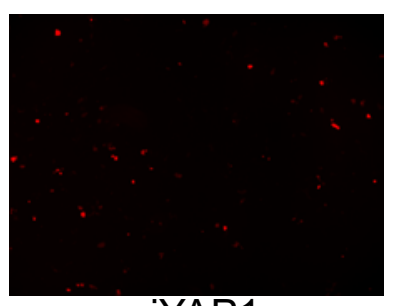

siYAP1

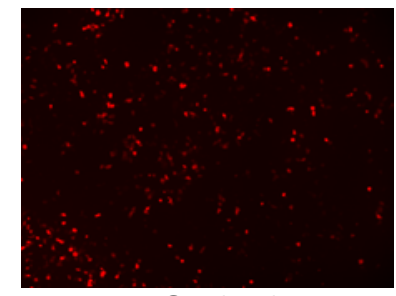

siSF3B3

D
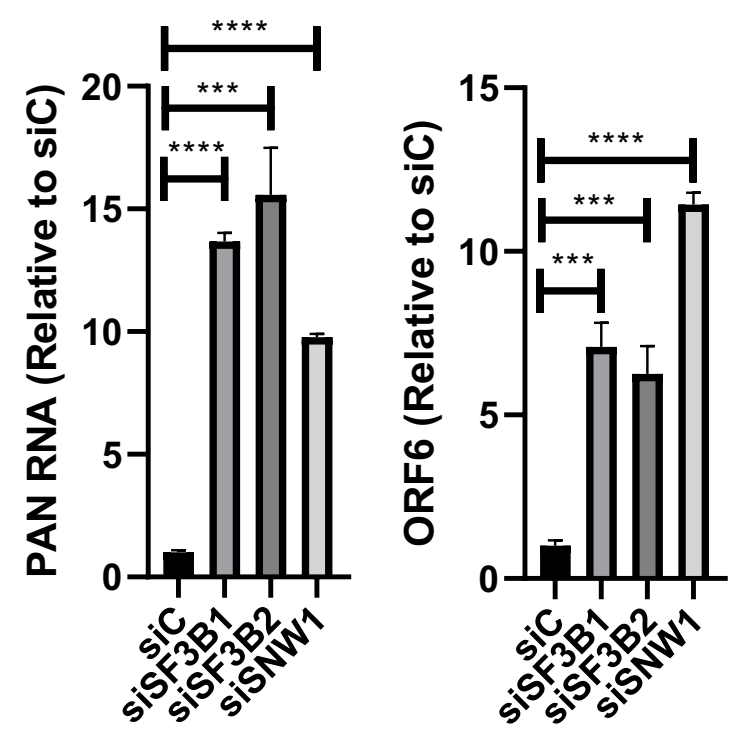

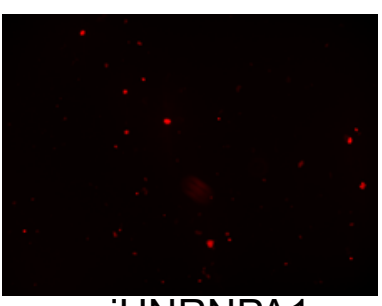

siHNRNPA1

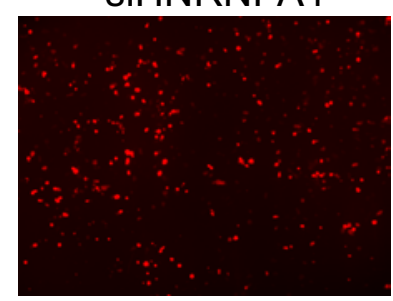

siSNW1

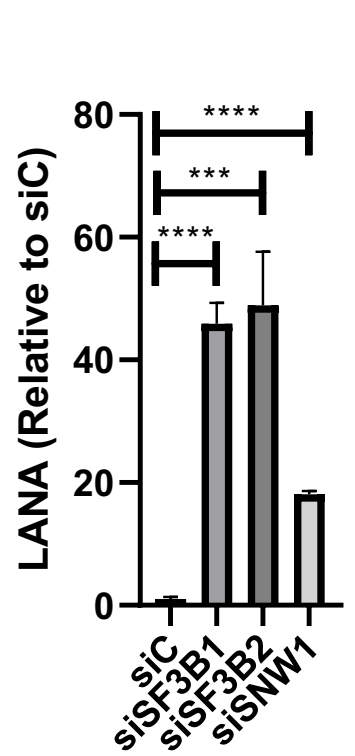

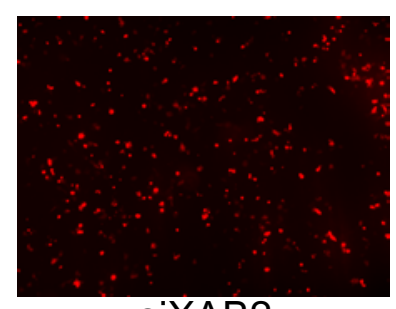

siXAB2

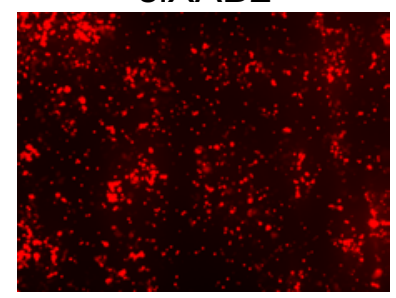

siCHD4

E

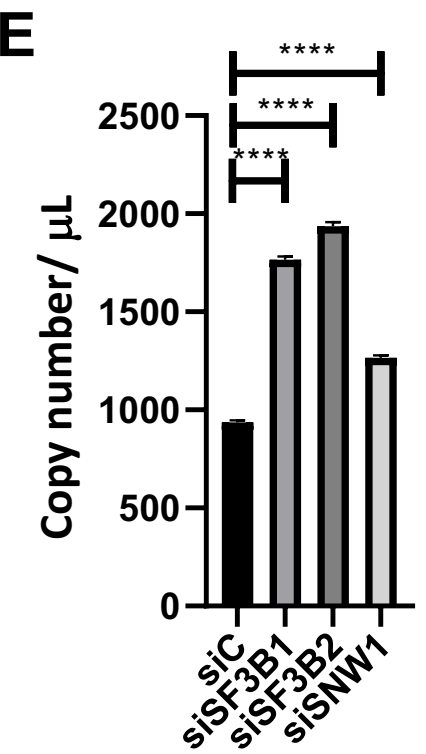


Fig. 6

(a)

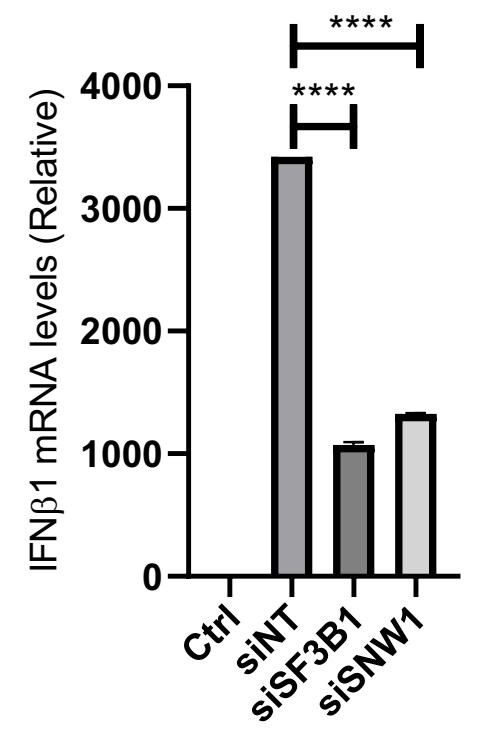

(d)

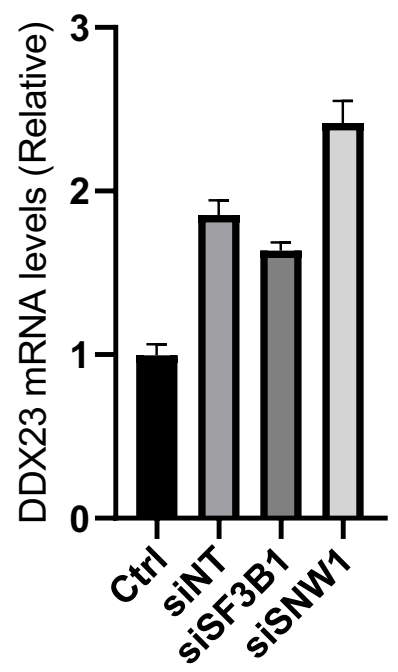

(b)

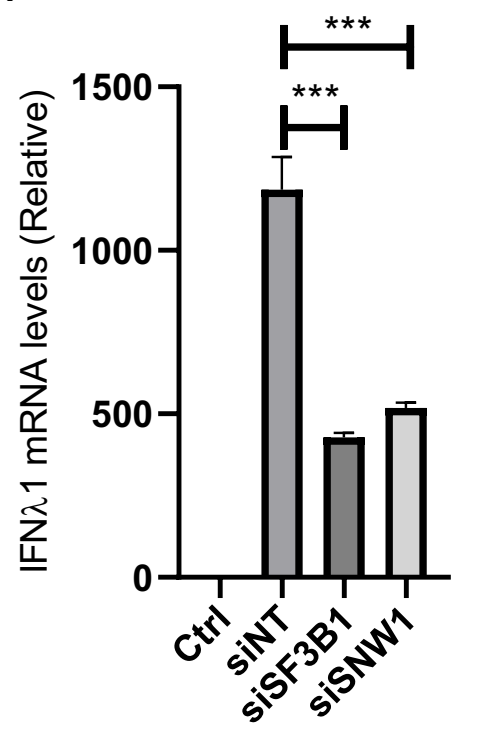

(c)

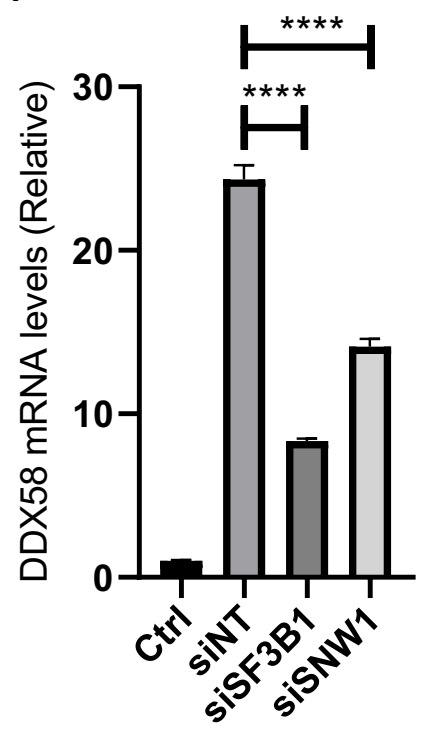

